\title{
SECOND RICCI FLOW ON NONCOMPACT HERMITIAN MANIFOLDS
}

\author{
MAN-CHUN LEE
}

\begin{abstract}
In this work, we first establish short time existence and Shi's type estimate of second Ricci flow on complete noncompact Hermitian manifolds. As an application, we use the second Ricci flow to discuss the existence of Kähler-Einstein metric on complete noncompact Hermitian manifolds.
\end{abstract}

\section{INTRODUCTION}

Let $(M, g, J)$ be a complete complex manifold and $g$ is a Hermitian metric. $g$ is Hermitian if $g$ is a Riemannian metric and also satisfies $g(X, Y)=$ $g(J X, J Y)$ for all $X, Y \in T M$. When $M$ is compact Kähler or complete noncompact Kähler with suitable curvature, it was shown that the Hamilton Ricci flow will preserve the Kähler condition [4, 25, 10]. The Kähler-Ricci flow was then found to be very powerful in the study of geometrical classification in Kähler geometry. However, when $g$ is non-Kähler, generally the Ricci flow will no longer preserve the Hermitian condition. Inspired by this, one may ask if there is any alternative parabolic flow which also preserves the Hermitian structure. In [8], Gill introduced a Hermitian flow called the Chern-Ricci flow which aims to study the existence of Hermitian metric with flat Chern-Ricci curvature.

In this work, we are interested in special case of Hermitian flow on complete noncompact Hermitian manifolds which was first introduced by Streets and Tian [26]:

$$
\frac{\partial}{\partial t} g=-S_{g}, \quad g(0)=g_{0}
$$

where $S_{g}$ is the second Ricci curvature with respect to the Chern connection of $g$. This flow was also appeared in [18] which aim to study Hermitian Einstein metric on complex manifolds. In this article, we will call it the Hermitian Ricci flow. In fact, in [26], a more general Hermitian flow was introduced in which the direction of the deformation may involve torsion terms $Q(T)$. The Hermitian curvature flow was also found to be useful in the study of Hermitian geometry. In [27, they initiated a program of studying a particular choice of

Date: December 3, 2019.

2010 Mathematics Subject Classification. Primary 53C55; Secondary 53C44 .

Key words and phrases. Hermitian manifold, holomorphic bisectional curvature, KählerEinstein metric. 
$Q$ in which the flow preserves the pluriclosed condition. More recently Ustinovskiy [30] also showed that for a different choice of $Q$ the flow will preserve the nonnegativity of bisectional curvature which leads to an extensions of the classical Frankel conjecture to quasi-positive case. Motivated by the work in [17, 3], the author study the first Ricci curvature along the flows with $Q=0$ and use it to show that compact Hermitian manifolds with quasi-negative bisectional curvature have ample canonical line bundle. By the celebrated work of Yau and Aubin, [1, 35], it is equivalent to say that there is a Kähler-Einstein metric with negative scalar curvature.

In this article, we wish to study the existence of Kähler-Einstein metric on complete noncompact Hermitian manifolds. To extend the work in [14, we first develop some foundational results on the short-time existence of the Hermitian flow under some reasonable assumption. In particular, we have the following short-time existence result.

Theorem 1.1. Suppose $\left(M, g_{0}\right)$ is a complete noncompact Hermitian manifold with

$$
\sup _{M}|R m|+|T|^{2}+|\nabla T|<+\infty,
$$

then there is a short-time solution $g(t)$ on $M \times[0, \tau]$ to

$$
\partial_{t} g_{i \bar{j}}=-S_{i \bar{j}}, \quad g(0)=g_{0} .
$$

Moreover, the solution $g(t)$ has bounded Chern curvature and torsion on $[0, \tau]$.

Remark 1.1. For general $Q$, the corresponding Hermitian curvature will also admit short-time solution under the assumption made above which is a noncompact version of Streets-Tian's work [26]. In fact under boundedness of Chern curvature and Torsion, the boundedness on $\nabla T$ is equivalent to boundedness of Riemannian curvature $R m^{L}$. When $Q=0$, this can be replaced by existence of good exhaustion function on $M$. We refer readers to Theorem 6.1 for detailed statement.

Next, we wish to apply the flow to study existence of Kähler-Einstein metric on negatively curved Hermitian manifolds.In this work, we are interested in the case when a complete noncompact Hermitian manifold has quasi-negative curvature. Using the existence of the Hermitian Ricci flow together with Shitype estimates, we extend the result in [14] to complete noncompact case. Our main result is the following.

Theorem 1.2. Let $\left(M, g_{0}\right)$ be a complete noncompact Hermitian manifold with bounded Riemannian curvature, Chern curvature and torsion. Suppose $g_{0}$ has non-positive Chern bisectional curvature and quasi-negative first Ricci curvature, then $M$ supports a Kähler metric which maybe incomplete. Furthermore, if the first Ricci curvature is uniformly negative outside a compact set, then $M$ supports a complete Kähler-Einstein metric $g_{K E}=-$ Ric $\left(g_{K E}\right)$ with bounded curvature. 
It is unclear to the author whether $M$ supports a complete Kähler metric in the quasi-negative case. When $M$ is noncompact, the existence of complete Kähler-Einstein metric was studied by various authors, see for example 6 , 31] based on assumptions on the Ricci curvature and [32, 12] based on the negativity of holomorphic sectional curvature. Theorem 1.2 is different from the previous results since the Kählerity is a priori unknown.

The paper is organized as follows: In section 2, we recall some preliminary definitions and formula about the Chern connection. In section 3, we will derive evolution equations for the Hermitian Ricci flow. In section 4, we will derive some a-priori estimates for the Hermitian Ricci flow. In section 5, 6, we will prove the general short-time existence to Hermitian manifolds with bounded Riemannian curvature, Chern curvature and torsion. In section 7, 8, we will give a proof of Theorem 1.2 .

Acknowledgement: The author would also like to thank the referee for useful comments.

\section{Chern COnNECTiOn}

In this section, we collect some useful formulas for the Chern connection. Those materials can be found in [23]. Let $(M, g)$ be a Hermitian manifold. The Chern connection of $g$ is defined as follows: In local holomorphic coordinates $z^{i}$, for a vector field $X_{i} \partial_{i}$, where $\partial_{i}:=\frac{\partial}{\partial z^{i}}, \partial_{\bar{i}}=\frac{\partial}{\partial \bar{z}^{i}}$,

$$
\nabla_{i} X^{k}=\partial_{i} X^{k}+\Gamma_{i j}^{k} X^{j} ; \nabla_{\bar{i}} X^{k}=\partial_{i} X^{k} .
$$

For a $(1,0)$ form $a=a_{i} d z^{i}$,

$$
\nabla_{i} a_{j}=\partial_{i} a_{j}-\Gamma_{i j}^{k} a_{k} ; \nabla_{\bar{i}} a_{j}=\partial_{i} a_{j} .
$$

Here $\nabla_{i}:=\nabla_{\partial_{i}}$, etc. $\Gamma$ are the coefficients of $\nabla$, with

$$
\Gamma_{i j}^{k}=g^{k \bar{l}} \partial_{i} g_{j \bar{l}}
$$

Noted that Chern connection is a connection such that $\nabla g=\nabla J=0$ and the torsion has no $(1,1)$ component. The torsion of $g$ is defined to be

$$
T_{i j}^{k}=\Gamma_{i j}^{k}-\Gamma_{j i}^{k} \text {. }
$$

We remark that $g$ is Kähler if and only if $T=0$. Define the Chern curvature tensor of $g$ to be

$$
R_{i \overline{j k}}^{l}=-\partial_{j} \Gamma_{i k}^{l} .
$$

We raise and lower indices by using metric $g$. Direct computations show:

$$
\overline{R_{i \bar{j} k \bar{l}}}=R_{j \bar{i} i \bar{k}} .
$$

In this note, we will use $R m$ to denote curvature tensor with respect to the Chern-connection while $R m^{L}$ will denote the Riemannian curvature tensor.

The Chern-Ricci curvature is defined by

$$
R_{i \bar{j}}=g^{k \bar{l}} R_{i \bar{j} k \bar{l}}=-\partial_{i} \partial_{\bar{j}} \log \operatorname{det} g .
$$


Note that if $g$ is not Kähler, then $R_{i \bar{\jmath}}$ may not equal to $g^{k \bar{l}} R_{k \bar{l} i \bar{j}}=S_{i \bar{j}}$. In some content, Ric is sometimes called first Ricci curvature while $S$ is called the second Ricci.

Lemma 2.1. The commutation formulas for the Chern curvature are given by

$$
\begin{aligned}
{\left[\nabla_{i}, \nabla_{\bar{j}}\right] X^{l}=R_{i \bar{j} k}{ }^{l} X^{k}, } & {\left[\nabla_{i}, \nabla_{\bar{j}}\right] a_{k}=-R_{i \bar{j} k}{ }^{l} a_{l} ; } \\
{\left[\nabla_{i}, \nabla_{\bar{j}}\right] X^{\bar{l}}=-R_{i \bar{j}}{ }^{\bar{l}}{ }_{\bar{k}} X^{\bar{k}}, } & {\left[\nabla_{i}, \nabla_{\bar{j}}\right] a_{\bar{k}}=R_{i \bar{j}}{ }^{\bar{l}}{ }_{\bar{k}} a_{\bar{l}} . }
\end{aligned}
$$

When $g$ is not Kähler, the Bianchi identities maybe fail. The failure can be measured by the torsion tensor.

Lemma 2.2. In a holomorphic local coordinates, let $T_{i j \bar{k}}=g_{p \bar{k}} T_{i j}^{p}$, we have

$$
\begin{aligned}
R_{i \bar{j} k \bar{l}}-R_{k \bar{j} i \bar{l}} & =-\nabla_{\bar{j}} T_{i k \bar{l}}, \\
R_{i \bar{j} k \bar{l}}-R_{i \bar{l} k \bar{j}} & =-\nabla_{i} T_{\bar{j} \bar{l} k}, \\
R_{i \bar{j} k \bar{l}}-R_{k \bar{l} i \bar{j}} & =-\nabla_{\bar{j}} T_{i k \bar{l}}-\nabla_{k} T_{\bar{j} \bar{l} i}=-\nabla_{i} T_{\bar{j} \bar{l} k}-\nabla_{\bar{l}} T_{i k \bar{j}}, \\
\nabla_{p} R_{i \bar{j} k \bar{l}}-\nabla_{i} R_{p \bar{j} k \bar{l}} & =-T_{p i}^{r} R_{r \bar{j} k \bar{l}}, \\
\nabla_{\bar{q}} R_{i \bar{j} k \bar{l}}-\nabla_{\bar{j}} R_{i \bar{q} k \bar{l}} & =-T_{\bar{q} \bar{j}}^{\bar{s}} R_{i \bar{s} k \bar{l}} .
\end{aligned}
$$

It can be checked easily that for $X, Y \in T^{1,0} M, R(X, \bar{X}, Y, \bar{Y})$ is real-valued. We consider the following curvature condition.

Definition 2.1. We say that $(M, g)$ has holomorphic bisectional curvature bounded above by a function $\kappa(x)$ if for any $x \in M, X, Y \in T_{x}^{1,0} M$,

$$
R(X, \bar{X}, Y, \bar{Y}) \leq \kappa B(X, \bar{X}, Y, \bar{Y})
$$

where $B_{i \bar{j} k \bar{l}}=g_{i \bar{j}} g_{k \bar{l}}+g_{i \bar{l}} g_{k \bar{j}}$.

Here we should remark that our notation of bisectional curvature is slightly different from that in [16].

Definition 2.2. We say that $(M, g)$ has Chern-Ricci curvature bounded above by a function $\kappa(x)$ if for any $p \in M, X \in T_{p}^{1,0} M$,

$$
\operatorname{Ric}(X, \bar{X}) \leq \kappa(p) g(X, \bar{X}) .
$$

If $\kappa$ is non-positive and negative at some point $z \in M$, then we say that $g$ has quasi-negative Chern-Ricci curvature.

In this note, all the curvature tensor $R m$ will be referring to the curvature tensor with respect to Chern connection.

\section{Evolution equations for the Hermitian Ricci flow}

In this section, we will discuss a special type of Hermitian Ricci flow introduced by [26] with $Q \equiv 0$ :

$$
\left\{\begin{array}{l}
\frac{\partial}{\partial t} g_{i \bar{\jmath}}=-S_{i \bar{j}} \\
g(0)=g_{0}
\end{array}\right.
$$


Here $S_{i \bar{j}}=g^{k \bar{l}} R_{k \bar{l} i \bar{j}}$ is the second Ricci curvature with respect to the Chern connection while the Chern-Ricci curvature (or first Ricci curvature) is defined by $R_{i \bar{j}}=g^{k \bar{l}} R_{i \bar{j} k \bar{l}}$. It coincides with the Chern-Ricci curvature if the metric is Kähler. However they are different in general.

To begin with, we would like to point out that the Hermitian Ricci flow is indeed a parabolic system which is in a similar form as the Ricci DeTurck flow which was shown explicitly by Shi in [24, Lemma 2.1].

Lemma 3.1. In local coordinate, we have

$$
\begin{aligned}
\frac{\partial}{\partial t} g_{i \bar{j}}= & \frac{1}{2} g^{k \bar{l}}\left(\tilde{\nabla}_{k} \tilde{\nabla}_{\bar{l}}+\tilde{\nabla}_{\bar{l}} \tilde{\nabla}_{k}\right) g_{i \bar{j}}-g^{k \bar{l}} g^{p \bar{q}}\left(\tilde{\nabla}_{k} g_{i \bar{q}}\right)\left(\tilde{\nabla}_{\bar{l}} g_{p \bar{j}}\right) \\
& -\frac{1}{2}\left(g^{k \bar{l}} g_{p \bar{j}} \tilde{R}_{k \bar{l} \bar{l}}^{p}+g^{k \bar{l}} g_{i \bar{q}} \tilde{R}_{k \bar{l}} \overline{\bar{q}}\right) .
\end{aligned}
$$

Here $\tilde{\nabla}$ and $\tilde{R}$ denotes the Chern connection and the Chern curvature of $g_{0}$ respectively.

Proof.

$$
\begin{aligned}
-S_{i \bar{j}}= & -g^{k \bar{l}} R_{k \bar{l} i \bar{j}} \\
= & g^{k \bar{l}} g_{p \bar{j}}\left(\partial_{\bar{l}} \Gamma_{k i}^{p}-\partial_{\bar{l}} \tilde{\Gamma}_{k i}^{p}\right)-g^{k \bar{l}} g_{p \bar{j}} \tilde{R}_{k \overline{l i}}{ }^{p} \\
= & g^{k \bar{l}} g_{p \bar{j}} \tilde{\nabla}_{\bar{l}}\left(g^{p \bar{q}} \tilde{\nabla}_{k} g_{i \bar{q}}\right)-g^{k \bar{l}} g_{p \bar{j}} \tilde{R}_{k \overline{l i}}^{p} \\
= & g^{k \bar{l}} \tilde{\nabla}_{\bar{l}} \tilde{\nabla}_{k} g_{i \bar{j}}-g^{k \bar{l}} g^{p \bar{q}}\left(\tilde{\nabla}_{k} g_{i \bar{q}}\right)\left(\tilde{\nabla}_{\bar{l}} g_{p \bar{j}}\right)-g^{k \bar{l}} g_{p \bar{j}} \tilde{R}_{k \overline{l i}} p \\
= & \frac{1}{2} g^{k \bar{l}}\left(\tilde{\nabla}_{k} \tilde{\nabla}_{\bar{l}}+\tilde{\nabla}_{\bar{l}} \tilde{\nabla}_{k}\right) g_{i \bar{j}}-g^{k \bar{l}} g^{p \bar{q}}\left(\tilde{\nabla}_{k} g_{i \bar{q}}\right)\left(\tilde{\nabla}_{\bar{l}} g_{p \bar{j}}\right) \\
& -\frac{1}{2}\left(g^{k \bar{l}} g_{p \bar{j}} \tilde{R}_{k \overline{l i}}^{p}+g^{k \bar{l}} g_{i \bar{q}} \tilde{R}_{k \bar{l}} \overline{\bar{q}}\right) .
\end{aligned}
$$

Lemma 3.2. Suppose $g(t)$ is a solution to the Hermitian Ricci flow, then we have

$$
\left(\frac{\partial}{\partial t}-\Delta\right) t r_{g} g_{0}=-\left(g_{0}\right)_{p \bar{q}} g^{k \bar{l}} g^{i \bar{j}} \Psi_{k i}^{p} \Psi_{\overline{l j}}^{\bar{q}}+g^{k \bar{l}} g^{i \bar{j}} \hat{R}_{k \bar{l} \bar{j}}
$$

where $\Psi=\Gamma_{g_{0}}-\Gamma_{g}$ denotes the difference between the Chern connection of $h$ and that of $g$ while $\hat{R}$ is the Chern curvature of $g_{0}$.

Proof. Differentiate it with respect to $t$, we have

$$
\frac{\partial}{\partial t}\left(g^{i \bar{j}}\left(g_{0}\right)_{i \bar{j}}\right)=S^{i \bar{j}}\left(g_{0}\right)_{i \bar{j}}
$$

On the other hand,

$$
\Delta\left(g^{i \bar{j}}\left(g_{0}\right)_{i \bar{j}}\right)=h_{p \bar{q}} g^{k \bar{l}} g^{i \bar{j}} \Psi_{k i}^{p} \Psi_{\bar{l} \bar{j}}^{\bar{q}}-g^{k \bar{l}} g^{i \bar{j}} \hat{R}_{k \bar{l} i \bar{j}}+S^{i \bar{j}} h_{i \bar{j}} .
$$

The conclusion follows immediately by adding (3.5) and (3.6) together. 
Lemma 3.3. Suppose $(M, g(t))$ is a soliution to (3.1), then the tensor $\Psi_{i j}^{k}=$ $\tilde{\Gamma}_{i j}^{k}-\Gamma_{i j}^{k}$ satisfies

$$
\left(\frac{\partial}{\partial t}-\Delta\right)|\Psi|^{2}=-|\nabla \Psi|^{2}-|\bar{\nabla} \Psi|^{2}+2 \operatorname{Re}\left[g^{i \bar{j}} g^{k \bar{l}} g_{r \bar{s}} \Psi_{{ }_{j} \bar{s}}^{\bar{s}}\left(g^{p \bar{q}} T_{p i}^{a} R_{a \bar{q} k}{ }^{r}+g^{p \bar{q}} \nabla_{p} \tilde{R}_{i \bar{q} k}{ }^{r}\right)\right] .
$$

Here $\tilde{\Gamma}$ and $\tilde{R}$ denotes the Chern connection and Chern curvature with respect to $g_{0}$ and the norm is calculated using the evolving metric $g(t)$.

Proof. First noted that

$$
\partial_{t} \Psi_{i j}^{k}=g^{k \bar{l}} \nabla_{i} S_{j \bar{l}}
$$

On the other hand,

$$
\begin{aligned}
\Delta|\Psi|^{2}= & g^{p \bar{q}} \nabla_{p} \nabla_{\bar{q}}\left(g^{i \bar{j}} g^{k \bar{l}} g_{r \bar{s}} \Psi_{i k}^{r} \Psi_{\bar{j} \bar{l}}^{\bar{s}}\right) \\
= & g^{p \bar{q}} g^{i \bar{j}} g^{k \bar{l}} g_{r \bar{s}} \nabla_{p} \nabla_{\bar{q}}\left(\Psi_{i k}^{r} \Psi_{\bar{j} \bar{l}}^{\bar{s}}\right) \\
= & g^{p \bar{q}} g^{i \bar{j}} g^{k \bar{l}} g_{r \bar{s}}\left(\nabla_{p} \Psi_{i k}^{r} \cdot \nabla_{\bar{q}} \Psi_{\bar{j} \bar{l}}^{\bar{s}}+\nabla_{\bar{q}} \Psi_{i k}^{r} \cdot \nabla_{p} \Psi_{\bar{j} \bar{l}}^{\bar{s}}\right) \\
& +g^{p \bar{q}} g^{i \bar{j}} g^{k \bar{l}} g_{r \bar{s}}\left(\nabla_{p} \nabla_{\bar{q}} \Psi_{i k}^{r} \cdot \Psi_{\bar{j}_{\bar{l}}}^{\bar{s}}+\nabla_{p} \nabla_{\bar{q}} \Psi_{\bar{j} \bar{l}}^{\bar{s}} \cdot \Psi_{i k}^{r}\right) \\
= & |\nabla \Psi|^{2}+|\bar{\nabla} \Psi|^{2}+g^{p \bar{q}} g^{i \bar{j}} g^{k \bar{l}} g_{r \bar{s}}\left(\nabla_{p} \nabla_{\bar{q}} \Psi_{i k}^{r} \cdot \Psi_{\bar{j} \bar{l}}^{\bar{s}}+\nabla_{p} \nabla_{\bar{q}} \Psi_{\bar{j} \bar{l}}^{\bar{s}} \cdot \Psi_{i k}^{r}\right) \\
= & |\nabla \Psi|^{2}+|\bar{\nabla} \Psi|^{2}+g^{p \bar{q}} g^{i \bar{j}} g^{k \bar{l}} g_{r \bar{s}} \Psi_{\bar{j} \bar{l}}^{\bar{s}} \nabla_{p}\left(R_{i \bar{q} k}^{r}-\tilde{R}_{i \bar{q} k}^{r}\right) \\
& +g^{p \bar{q}} g^{i \bar{j}} g^{k \bar{l}} g_{r \bar{s}} \Psi_{i k}^{r} \bar{\nabla}_{q}\left(R_{j \bar{p} l}^{s}-\tilde{R}_{j \bar{p} l}^{s}\right) \\
& +g^{i \bar{j}} g^{k \bar{l}} g_{r \bar{s}} \Psi_{i k}^{r}\left(S^{\bar{s}}{ }_{\bar{q}} \Psi_{\bar{j} \bar{l}}^{\bar{q}}-S^{\bar{q}}{ }_{\bar{j}} \Psi_{\bar{q} \bar{l}}^{\bar{s}}-S^{\bar{q}}{ }_{\bar{l}} \Psi_{\bar{j} \bar{q}}^{\bar{s}}\right) \\
= & |\nabla \Psi|^{2}+|\bar{\nabla} \Psi|^{2}+2 R e\left[g^{i \bar{j}} g^{k \bar{l}} g_{r \bar{s}} \Psi_{\bar{j} \bar{l}}^{\bar{s}}\left(\nabla_{i} S_{k}^{r}-g^{p \bar{q}} T_{p i}^{a} R_{a \bar{q} k}^{r}-g^{p \bar{q}} \nabla_{p} \tilde{R}_{i \bar{q} k}{ }^{r}\right)\right] \\
& +g^{i \bar{j}} g^{k \bar{l}} g_{r \bar{s}} \Psi_{i k}^{r}\left(S^{\bar{s}}{ }_{\bar{q}} \Psi_{\bar{j} \bar{l}}^{\bar{q}}-S^{\bar{q}}{ }_{j} \Psi_{\bar{q} \bar{l}}^{\bar{s}}-S^{\bar{q}}{ }_{\bar{l}} \Psi_{\bar{j} \bar{q}}^{\bar{s}}\right)
\end{aligned}
$$

where we have used the fact that

$$
g^{p \bar{q}} \nabla_{p} R_{i \bar{q} k}^{r}=\nabla_{i} S_{k}^{r}-g^{p \bar{q}} T_{p i}^{s} R_{s \bar{q} k}^{r} .
$$

Therefore, we can conclude that

$$
\left(\frac{\partial}{\partial t}-\Delta\right)|\Psi|^{2}=-|\nabla \Psi|^{2}-|\bar{\nabla} \Psi|^{2}+2 \operatorname{Re}\left[g^{i \bar{j}} g^{k \bar{l}} g_{r \bar{s}} \Psi_{\bar{j} \bar{l}}^{\bar{s}}\left(g^{p \bar{q}} T_{p i}^{a} R_{a \bar{q} k}{ }^{r}+g^{p \bar{q}} \nabla_{p} \tilde{R}_{i \bar{q} k}{ }^{r}\right)\right]
$$

Now we collect the evolution equation for the Chern curvature tensor $R_{i \bar{j} k \bar{l}}$ which can be found in [26, Section 6]. 
Lemma 3.4. Suppose $g(t)$ is a solution to the Hermitian Ricci flow, we have

$$
\begin{aligned}
\partial_{t} R_{i \bar{j} k \bar{l}}= & \Delta R_{i \bar{j} k \bar{l}}+g^{r \bar{s}}\left[T_{r i}^{p} \nabla_{\bar{s}} R_{p \bar{j} k \bar{l}}+T_{\bar{s} \bar{j}}^{\bar{q}} \nabla_{r} R_{i \bar{q} k \bar{l}}+T_{r i}^{p} T_{\bar{s} \bar{j}}^{\bar{q}} R_{p \bar{q} k \bar{l}}\right. \\
& \left.+R_{i \bar{j} r}^{p} R_{p \bar{s} k \bar{l}}+R_{r \bar{j} k}^{p} R_{i \bar{s} p \bar{l}}-R_{r \bar{j} p \bar{l}} R_{i \bar{s} k}^{p}\right] \\
& -\frac{1}{2}\left[S_{i}^{p} R_{p \bar{j} k \bar{l}}+S_{k}^{p} R_{i \bar{j} p \bar{l}}+S_{\bar{j}}^{\bar{q}} R_{i \bar{q} k \bar{l}}+S_{\bar{l}}^{\bar{q}} R_{i \bar{j} k \bar{q}}\right] .
\end{aligned}
$$

By tracing $k$ and $l$, we arrive at the evolution equation of the Chern-Ricci curvature (or first Ricci curvature). For detailed computation, we refer to [14].

Lemma 3.5. Suppose $g(t)$ is a solution to the Hermitian Ricci flow, we have the following evolution equation for the Chern-Ricci curvature.

$$
\begin{aligned}
\partial_{t} R_{i \bar{j}}= & \Delta R_{i \bar{j}}+g^{r \bar{s}}\left(T_{r i}^{p} \nabla_{\bar{s}} R_{p \bar{j}}+T_{\bar{s} \bar{j}}^{\bar{q}} \nabla_{r} R_{i \bar{q}}+T_{r i}^{p} T_{\bar{s} \bar{j}}^{\bar{q}} R_{p \bar{q}}\right) \\
& +R_{i \bar{j} k}^{p} R_{p}^{k}-\frac{1}{2}\left[S_{i}^{p} R_{p \bar{j}}+S_{\bar{j}}^{\bar{q}} R_{i \bar{q}}\right] .
\end{aligned}
$$

We also have the following evolution equations for higher order derivative which is a sight modification of [26, Lemma 7.1-7.2].

Lemma 3.6. Suppose $g(t)$ is a solution to the Hermitian Ricci flow, then the Chern-curvature and the torsion of $g(t)$ satisfy the following equations.

$$
\begin{aligned}
\frac{\partial}{\partial t} \nabla^{k} R m= & \Delta \nabla^{k} R m+\sum_{j=0}^{k} \nabla^{j} T * \nabla^{k+1-j} R m+\sum_{j=0}^{k} \nabla^{j} R m * \nabla^{k-j} R m \\
& +\sum_{j=0}^{k} \sum_{l=0}^{j} \nabla^{l} T * \nabla^{j-l} T * \nabla^{k-j} R m, \\
\frac{\partial}{\partial t} \nabla^{k} T= & \Delta \nabla^{k} T+\sum_{j=0}^{k+1} \nabla^{j} T * \nabla^{k+1-j} T+\sum_{j=0}^{k} \nabla^{j} T * \nabla^{k-j} R m .
\end{aligned}
$$

Proof. The proof is identical to that in [26, Section 7] except now $Q \equiv 0$ and hence the last term in their formula vanishes.

\section{A PRIORI ESTIMATES}

In this section, we will establish some local estimates for the Hermitian Ricci flow $g(t)$ on compact subset. We first need some estimates on distance function.

Lemma 4.1. Suppose $\left(M^{n}, g\right)$ be a complete noncompact Hermitian manifold with complex dimension $n$ and $\mathrm{BK}_{g_{0}} \geq-K$ on $B_{g_{0}}(p, 2 r)$. Let $p \in M$ and $d_{g}(x, p)$ be the distance from $p$ with respect to $g$, then whenever $d_{g}(x, p) \in$ $\left[\frac{1}{\sqrt{K}}, r\right]$,

$$
\partial_{i} \partial_{\bar{j}} d_{g}(x, p) \leq C_{n} \sqrt{K} g_{i \bar{j}}
$$

within the cut-locus of $p$. 
Proof. Consider $\tilde{g}=K g$, then $|R m(\tilde{g})|+|\tilde{T}|^{2} \leq 1$ on $B_{\tilde{g}}(p, 2 \sqrt{K} r)$. We may apply [36, Theorem 1.1], although it is stated globally, one can check easily that the proof only requires bisectional curvature lower bound locally. Therefore, we have on $B_{\tilde{g}}(p, \sqrt{K} r) \backslash B_{\tilde{g}}(p, 1)$.

$$
\partial_{i} \partial_{\bar{j}} d_{\tilde{g}}(x, p) \leq C_{n} \sqrt{K} \tilde{g}_{i \bar{j}} .
$$

The result follows after we rescale it back to $g$.

Proposition 4.1. There is $\epsilon_{n}>0$ such that the following holds. Suppose $g(t)$ is a solution to (3.1) on $B_{g_{0}}(p, r+\delta) \times[0, \tau]$ for some $p \in M, r, \delta>0$. If the Hermitian Ricci flow solution $g(t)$ satisfies

$$
\left(1+\epsilon_{n}\right)^{-1} g_{0} \leq g(t) \leq\left(1+\epsilon_{n}\right) g_{0}
$$

on $B_{g_{0}}(p, \delta+r) \times[0, \tau]$. Let $K=\sup _{B_{g_{0}}(p, 2 r)} \sum_{i=0}^{1}\left|\nabla_{g_{0}}^{i} R m\left(g_{0}\right)\right|+\left|\nabla_{g_{0}}^{i} T_{g_{0}}\right|$, then there is $C(n, \delta, K)>0$ such that on $B_{g_{0}}(p, r) \times[0, \tau]$,

$$
|R m|+|\nabla T|+\left|\nabla_{g_{0}} g(t)\right| \leq C(n, \delta, K) .
$$

Proof. In what follows, we will use $C_{i}$ to denote any generic constant depending only on $n, r, K$. For notational convenience, we will use $\hat{R}, \hat{T}$ to denote the geometric quantities of $g_{0}$.

We first show the bound on $\left|\nabla_{g_{0}} g(t)\right|$. By our assumption and Lemma 3.2, the function $\Lambda=\operatorname{tr}_{g} g_{0}$ satisfies

$$
\left(\frac{\partial}{\partial t}-\Delta\right) \Lambda \leq-\frac{1}{1+\epsilon_{n}}|\Psi|^{2}+C_{0} .
$$

On the other hand, since we have

$$
\begin{aligned}
R_{i \bar{j} k}^{l} & =\hat{R}_{i \bar{j} k}{ }^{l}+\partial_{\bar{j}} \Psi_{i k}^{l} ; \\
T_{i j}^{k} & =\hat{T}_{i j}^{k}+\Psi_{i j}^{k}-\Psi_{j i}^{k} .
\end{aligned}
$$

Therefore, together with Lemma 3.3, the function $|\Psi|^{2}$ where $\Psi=\Gamma_{g_{0}}-\Gamma_{g(t)}$ satisfies

$$
\begin{aligned}
\left(\frac{\partial}{\partial t}-\Delta\right)|\Psi|^{2} \leq & -|\nabla \Psi|^{2}-|\bar{\nabla} \Psi|^{2} \\
& +2 \operatorname{Re}\left[g^{p \bar{q}} g^{i \bar{j}} g^{k \bar{l}} g_{r \bar{s}} \Psi_{\bar{j} \bar{l}}^{\bar{s}}\left(T_{p i}^{a} R_{a \bar{q} k}{ }^{r}+\nabla_{p} \hat{R}_{i \bar{q} k}{ }^{r}\right)\right] \\
\leq & -\frac{1}{2}\left(|\nabla \Psi|^{2}+|\bar{\nabla} \Psi|^{2}\right)+8|\Psi|^{4}+C_{1}
\end{aligned}
$$

Let $G(s)=e^{A s}+B$ where $A$ and $B$ are some positive constants to be specified. Let $\phi(s)$ be a cutoff function on $[0,+\infty)$ such that $\phi \equiv 1$ on $[0, r+$ $\delta / 2]$, vanishes outside $[0, r+\delta]$ and satisfies

$$
\left|\phi^{\prime}\right|^{2} \leq 100 \delta^{-2} \phi, \phi^{\prime \prime} \geq-100 \phi \delta^{-2} \text {. }
$$

Define $\Phi(x)=\phi\left(d_{g_{0}}(x, p)\right)$ to be a cutoff function on $M$ where $d_{g_{0}}(x, p)$ is the distance from the fixed point $p$ using Hermitian metric $g_{0}$. By Lemma 4.1 
and the trick of Calabi, we may assume $d_{g_{0}}(x, p)$ to be smooth and satisfy $\sqrt{-1} \partial \bar{\partial} d_{0}(x, p) \leq C_{3}(n, K) \omega_{g_{0}}$ when we apply maximum principle.

Consider the function $F(x, t)=|\Psi|^{2} G(\Lambda)$ on $M \times[0, \tau]$. Then on $B_{g_{0}}(p, r+$ $\delta) \times[0, \tau]$, it satisfies

$$
\begin{aligned}
\left(\frac{\partial}{\partial t}-\Delta\right) F= & G\left(\frac{\partial}{\partial t}-\Delta\right)|\Psi|^{2}+|\Psi|^{2}\left(\frac{\partial}{\partial t}-\Delta\right) G \\
& -2 \operatorname{Re}\left(\Phi g^{i \bar{j}} G_{\bar{j}} \cdot \partial_{i}|\Psi|^{2}\right) \\
\leq & -\frac{1}{2}\left(|\nabla \Psi|^{2}+|\bar{\nabla} \Psi|^{2}\right)\left(e^{A \Lambda}+B\right) \\
& +|\Psi|^{4}\left[8\left(e^{A \Lambda}+B\right)-\frac{A}{1+\epsilon_{n}} e^{A \Lambda}\right] \\
& -A|\Psi|^{2}|\nabla \Lambda|^{2} e^{A \Lambda}+2 e^{A \Lambda} A|\nabla \Lambda||\Psi||\nabla \Psi|+C_{4} .
\end{aligned}
$$

If we choose $B=10 e^{A n\left(1+\epsilon_{n}\right)}$, then we can use Cauchy inequality to simplify it as

$$
\begin{aligned}
\left(\frac{\partial}{\partial t}-\Delta\right) F \leq & -\frac{1}{4}\left(|\nabla \Psi|^{2}+|\bar{\nabla} \Psi|^{2}\right)\left(e^{A \Lambda}+B\right)+C_{5} \\
& +\frac{1}{1+\epsilon_{n}}|\Psi|^{4}\left[90 e^{A n\left(1+\epsilon_{n}\right)}-A e^{A n\left(1-\epsilon_{n}\right)}\right] .
\end{aligned}
$$

Hence, we can choose $A$ sufficiently large such that

$$
100 e^{A n\left(1+\epsilon_{n}\right)}-A e^{A n\left(1-\epsilon_{n}\right)}<0
$$

provided that $\epsilon_{n}$ is small enough. With this choice of $A, B$ and $\epsilon_{n}, F$ satisfies

$$
\left(\frac{\partial}{\partial t}-\Delta\right) F \leq-c_{6} F^{2}+C_{7} \text {. }
$$

Using (4.7), we can apply maximum principle on function $F \cdot \Phi$. If the maximum is attained at $t=0$, then the conclusion is trivially true. Suppose it is attained at $t=t_{0}>0$, then

$$
\begin{aligned}
0 & \leq\left(\frac{\partial}{\partial t}-\Delta\right)(F \Phi) \\
& \leq \Phi\left(\frac{\partial}{\partial t}-\Delta\right) F+F\left(\frac{\partial}{\partial t}-\Delta\right) \Phi-2 \operatorname{Re}\left(g^{i \bar{j}} F_{i} \Phi_{\bar{j}}\right) \\
& \leq-c_{6} F^{2} \Phi+C_{8}+F\left(C_{8} \delta^{-2}+C_{8}\right)
\end{aligned}
$$

which implies $F \Phi$ is bounded above by $C_{9}\left(r^{-4}+1\right)$ at its maximum point. In particular, on $B_{g_{0}}(p, r+\delta / 2) \times[0, \tau]$,

$$
|\Psi|^{2} \leq C_{10}\left(\delta^{-4}+1\right) .
$$

This shows the bound on $\left|\nabla_{g_{0}} g(t)\right|$. 
For $|R m|$ and $|\nabla T|$, the proof is similar but simpler. By Lemma 3.6 with Cauchy inequality, the function $G=\sqrt{|R m|^{2}+|\nabla T|^{2}}$ satisfies

$$
\left(\frac{\partial}{\partial t}-\Delta\right) G \leq C_{n} G^{2}+C_{n}
$$

whenever $|R m|^{2}+|\nabla T|^{2} \neq 0$.

On the other hand, since we have established the estimate on $\Psi$, (4.4) have the following form now.

$$
\left(\frac{\partial}{\partial t}-\Delta\right)|\Psi|^{2} \leq-\frac{1}{2}\left(|\nabla \Psi|^{2}+|\bar{\nabla} \Psi|^{2}\right)+C_{9}
$$

on $B_{g_{0}}(p, r+\delta / 2) \times[0, \tau]$. Because

$$
\begin{aligned}
& \bar{\nabla} \Psi=R m(g(t))-R m\left(g_{0}\right) ; \\
& \nabla T=\nabla\left(T-T_{g_{0}}\right)+\Psi * T_{g_{0}}=\nabla \Psi+\Psi * T_{g_{0}},
\end{aligned}
$$

we can rewrite (4.4) to be

$$
\left(\frac{\partial}{\partial t}-\Delta\right)|\Psi|^{2} \leq-c_{10} G^{2}+C_{11}
$$

Then for $L(n, K, \delta)$ sufficiently large, the function $H=G+L|\Psi|^{2}$ satisfies

$$
\left(\frac{\partial}{\partial t}-\Delta\right) H \leq-H^{2}+C_{12}
$$

Therefore, we may use cutoff function trick again as (4.8) to show the bound on $B_{g_{0}}(p, r) \times[0, \tau]$.

Proposition 4.2. Suppose $g(t)$ is a solution to (3.1) on $B_{g_{0}}(p, r+\delta) \times[0, \tau]$ for some $p \in M, r, \delta>0$. If the Hermitian Ricci flow solution $g(t)$ satisfies

$$
|R m(g(t))|+|T|^{2} \leq K_{0}
$$

on $B_{g_{0}}(p, r+\delta) \times[0, \tau]$ for some $K_{0}>0$. Let $B_{m}$ be such that

$$
\sup _{B_{g_{0}}(p, r+\delta)} \sum_{i=0}^{m}\left|\nabla^{i} R m\left(g_{0}\right)\right|+\left|\nabla_{g_{0}}^{i} T\left(g_{0}\right)\right| \leq B_{m} .
$$

Then for any $m \in \mathbb{N}$, there is $C\left(n, m, \delta, B_{m}, K_{0}\right)>0$ such that on $B_{g_{0}}(p, r+$ $\left.\frac{\delta}{m+1}\right) \times[0, \tau]$,

$$
\left|\nabla^{m} R m\right|+\left|\nabla^{m} T\right| \leq C
$$

Proof. The proof is similar to that in Proposition 4.3. We prove the assertion by induction on $m$. In the proof, we will denote any generic constant depending only on $n, m, \delta, B_{m}, K_{0}$ by $C_{i}$. Assumption implies that the conclusion is true 
for $m=0$. Assume it is true for $m=0,1, \ldots, k-1$ for some $k \in \mathbb{N}$. By Lemma 3.6 and the induction hypothesis, the function $H_{i}=\left|\nabla^{i} R m\right|^{2}+\left|\nabla^{i} T\right|^{2}$ satisfies

$$
\begin{aligned}
\left(\frac{\partial}{\partial t}-\Delta\right) H_{k-1} & \leq-\frac{1}{2} H_{k}+C_{1} \\
\left(\frac{\partial}{\partial t}-\Delta\right) H_{k} & \leq-\frac{1}{2} H_{k+1}+C_{1} H_{k}+C_{1} .
\end{aligned}
$$

Define the function $H=H_{k}\left(H_{k-1}+A\right)$ where $A$ is some large constant to be specified later. Then

$$
\begin{aligned}
\left(\frac{\partial}{\partial t}-\Delta\right) H \leq & H_{k}\left(\frac{\partial}{\partial t}-\Delta\right) H_{k-1}+\left(A+H_{k-1}\right)\left(\frac{\partial}{\partial t}-\Delta\right) H_{k} \\
& -2 \operatorname{Re}\left(g^{i \bar{j}} \partial_{i} H_{k} \cdot \partial_{j} H_{k-1}\right) \\
\leq & -\frac{1}{2} H_{k}^{2}+C_{2} H_{k}+C_{2} H_{k} \sqrt{H_{k+1} H_{k-1}} \\
& +\left(A+H_{k-1}\right)\left[-\frac{1}{2} H_{k+1}+C_{1}+C_{1} H_{k}\right]
\end{aligned}
$$

where we have used the fact that $\left|\nabla H_{i}\right| \leq \sqrt{H_{i} H_{i+1}}$. By Cauchy inequality again, if $A$ is sufficiently large, then

$$
\begin{aligned}
\left(\frac{\partial}{\partial t}-\Delta\right) H & \leq H_{k}^{2}\left(-\frac{1}{2}+\frac{C_{n}}{A}\right)+C_{3} H_{k}+C_{3} \\
& \leq-\frac{1}{4} H_{k}^{2}+C_{3} H_{k}+C_{3} \\
& \leq-c_{4} H^{2}+C_{5}
\end{aligned}
$$

on $B_{g_{0}}(p, r+\delta / k) \times[0, \tau]$. Now the evolution equation is in the standard form. Let $d_{g_{0}}(x, p)$ and $\Phi=\phi\left(d_{g_{0}}(x, p)\right)$ where $\phi$ is a cutoff function on $[0,+\infty)$ such that $\phi \equiv 1$ on $\left[0, r+\frac{\delta}{k+1}\right]$, vanishes outside $\left[0, r+\frac{\delta}{k}\right]$ and satisfies $\left|\phi^{\prime}\right|^{2} \leq C_{6} \phi$ and $\phi^{\prime \prime} \geq-C_{6} \phi$. By our assumption, $g(t)$ is uniformly equivalent to $g_{0}$. Together with Lemma 4.1, we conclude that if the function $H \cdot \Phi$ achieves its maximum at $\left(x_{0}, t_{0}\right)$ where $t_{0}>0$, then

$$
\left(\frac{\partial}{\partial t}-\Delta\right)(H \Phi) \leq-c_{4} H^{2} \Phi+C_{6} H+C_{6} .
$$

By maximum principle, $H_{k} \leq C_{7}$ on $B_{g_{0}}\left(p, r+\frac{\delta}{k}\right) \times[0, \tau]$.

In fact, the higher order derivatives of $R m$ and $T$ will be instantly bounded after $g(t)$ evolves.

Proposition 4.3. Suppose $g(t)$ is a solution to (3.1) on $B_{g_{0}}(p, r+\delta) \times[0, \tau]$ for some $p \in M, r, \delta>0$. If the Hermitian Ricci flow solution $g(t)$ satisfies

$$
|R m|+|T|^{2} \leq K_{0}
$$


on $B_{g_{0}}(p, r+\delta) \times[0, \tau]$ for some $K_{0}>0$. Then for any $m \in \mathbb{N}$, there is $C_{0}\left(n, m, \tau, \delta, K_{0}\right)>0$ such that on $B_{g_{0}}\left(p, r+\frac{\delta}{m+1}\right) \times[0, \tau]$,

$$
\left|\nabla^{m} R m\right|+\left|\nabla^{m} T\right| \leq C_{0} t^{-m / 2} .
$$

Proof. In what follows, we will use $C_{i}$ to denote any generic constants depending only on $n, m, S, \delta, K_{0}$. We prove the assertion by induction on $m$. Assumption ensures that it is true when $m=0$. Assume it is true when $m=0,1, \ldots, k-1$ for some $k \in \mathbb{N}$. Let $G_{i}=t^{i} H_{i}$ where $H_{i}$ is defined in the exactly same way as in the proof of Proposition 4.2. By Lemma 3.6 and the induction hypothesis,

$$
\begin{aligned}
\left(\frac{\partial}{\partial t}-\Delta\right) G_{k-1} & \leq-\frac{1}{2} G_{k} t^{-1}+C_{1} t^{-1} \\
\left(\frac{\partial}{\partial t}-\Delta\right) G_{k} & \leq-\frac{1}{2} G_{k+1} t^{-1}+C_{1}+C_{1} G_{k} t^{-1} .
\end{aligned}
$$

Consider the new function $G=G_{k}\left(A+G_{k-1}\right)$. Argue as in the proof of Proposition 8.2

$$
\begin{aligned}
\left(\frac{\partial}{\partial t}-\Delta\right) G & \leq G_{k}^{2} t^{-1}\left(-\frac{1}{2}+\frac{C_{n}}{A}\right)+C_{3} G_{k} t^{-1}+C_{3} \\
& \leq-\frac{1}{4} G_{k}^{2} t^{-1}+C_{3} G_{k} t^{-1}+C_{3} \\
& \leq-c_{4} G^{2} t^{-1}+C_{5} G t^{-1}+C_{5}
\end{aligned}
$$

on $B_{g_{0}}(p, r+\delta / k)$ provided that $A$ is sufficiently large.

Let $\phi(s)$ be a cutoff function on $[0,+\infty)$ such that $\phi \equiv 1$ on $\left[0, r+\frac{\delta}{k+1}\right]$, vanishes outside $[0, r+\delta / k]$ and satisfies

$$
\left|\phi^{\prime}\right|^{2} \leq C_{6} \phi, \phi^{\prime \prime} \geq-C_{6} \phi .
$$

Define $\Phi(x)=\phi\left(d_{g_{0}}(x, p)\right)$ to be a cutoff function on $M$ where $d_{g_{0}}(x, p)$ is the distance from the fixed point $p$ using the Hermitian metric $g_{0}$. By (4.19), flow equation (3.1) and Lemma 4.1, if the function $F=\Phi G$ attains its maximum at $\left(x_{0}, t_{0}\right)$ where $t_{0}>0$, then at this point

$$
\begin{aligned}
0 & \leq\left(\frac{\partial}{\partial t}-\Delta\right) F \\
& \leq \Phi\left(\frac{\partial}{\partial t}-\Delta\right) G+G\left(\frac{\partial}{\partial t}-\Delta\right) \Phi+2 G \frac{|\nabla \Phi|^{2}}{\Phi} \\
& =-c_{4} F^{2} \Phi^{-1} t^{-1}+C_{7} F \Phi^{-1} t^{-1}+C_{7} .
\end{aligned}
$$

Hence, $F$ is bounded from above by some constant $C_{7}$ at this point and hence on $M \times[0, \tau]$. If $t_{0}=0$, then the conclusion trivially holds. Hence, the statement is true for $m=k$. By induction, this completes the proof. 


\section{Short-time EXISTEnCE Under Bounded GeOMETRY}

In this section, we consider the short time existence to (3.1) on complete noncompact Hermitian manifolds with bounded geometry of infinity order. Let us first recall the definition of bounded geometry:

Definition 5.1. Let $\left(M^{n}, g\right)$ be a complete Hermitian manifold. Let $k \geq 1$ be an integer and $0<\alpha<1 . g$ is said to have bounded geometry of order $k+\alpha$ if there are positive numbers $r, \kappa_{1}, \kappa_{2}$ such that at every $p \in M$ there is a neighborhood $U_{p}$ of $p$, and local biholomorphism $\xi_{p}$ from $D(r)$ onto $U_{p}$ with $\xi_{p}(0)=p$ satisfying the following properties:

(i) the pull back metric $\xi_{p}^{*}(g)$ satisfies:

$$
\kappa_{1} g_{e} \leq \xi_{p}^{*}(g) \leq \kappa_{2} g_{e}
$$

where $g_{e}$ is the standard metric on $\mathbb{C}^{n}$;

(ii) the components $g_{i \bar{\jmath}}$ of $\xi_{p}^{*}(g)$ in the natural coordinate of $D(r) \subset \mathbb{C}^{n}$ are uniformly bounded in the standard $C^{k+\alpha}$ norm in $D(r)$ independent of $p$.

$(M, g)$ is said to have bounded geometry of infinity order if instead of (ii) we have for any $k$, the $k$-th derivatives of $g_{i j}$ in $D(r)$ are bounded by a constant independent of $p . g$ is said to have bounded geometry of infinite order on a compact set $\Omega$ if (i) and (ii) are true for all $k$ for all $p \in \Omega$.

From Lemma 3.1, we see that the Hermitian Ricci flow equation is strongly parabolic if $g(t)$ is uniformly equivalent to some fixed metric, say for example $g_{0}$. Moreover, we can freely replace the Chern connection in Lemma 3.1 by the Levi-Civita connection since $g_{0}$ is assumed to have bounded geoemtry of infinity order. The short-time existence result will then follow by a standard inverse function theorem argument. For more details, we refer readers to [24, section 3-4], [13, Chapter VII, Theorem 7.1], [22, Section 4] and [2, Theorem 3.7.1].

Theorem 5.1. Let $\left(M, g_{0}\right)$ be a complete noncompact Hermitian manifold with bounded geometry of infinity order. Then there is $\tau\left(n, g_{0}\right)>0$ such that (3.1) has a solution on $M \times[0, \tau]$. Moreover, on $M \times[0, \tau]$, we have

$$
\left(1+\epsilon_{n}\right)^{-1} g_{0} \leq g(t) \leq\left(1+\epsilon_{n}\right) g_{0}
$$

where $\epsilon_{n}$ is the constant in Proposition 4.1.

Proof. Since $g_{0}$ has bounded geomtry of infinity order, we are free to interchange the Levi-Civita connection of $g_{0}$ and the Chern connection of $g_{0}$. Therefore by Lemma 3.1, the equation of the Hermitian Ricci flow has the form

$$
\left(\partial_{t}-g^{A B} \tilde{\nabla}_{A}^{L} \tilde{\nabla}_{B}^{L}\right) g(t)=Q\left(g, \tilde{\nabla}^{L} g\right)
$$

where $\tilde{\nabla}^{L}$ denotes the Levi-civita connection of $g_{0}$. Hence, it has exactly same form as the Deturck Ricci flow. Since we assume $g_{0}$ to have bounded geometry 
of infinity order, the proof of [2, Theorem 3.7.1] can be carried over (the first case in the proof). It is clear from the argument in [2, Theorem 3.7.1] that $g(t)$ can be as close to $g_{0}$ as we wish by shrinking the existence time.

\section{General Short-time existence on $M$}

In this section, we will show that one can construct a solution to 3.1 with uniformly bounded $|R m|+|T|^{2}$ if $g_{0}$ has bounded $\left|R m\left(g_{0}\right)\right|+\left|T_{g_{0}}\right|^{2}+\left|R m^{L}\left(g_{0}\right)\right|$. In the celebrated work by Shi 24], Shi showed that in fact the constructed solution of the DeTurck Ricci flow will have bounded curvature by establishing an integral estimate. And therefore, the curvature of the corresponding Ricci flow is uniformly bounded for a short time. For the Hermitian Ricci flow, the integral estimate is a bit tedious due to the presence of torsion. To bypass the complicated integration argument, we take an alternative path using the idea in [15]. By the work of [24, 29], there is an exhaustion function $\rho$ with $|\partial \rho|^{2}+|\sqrt{-1} \partial \bar{\partial} \rho| \leq C$ if $g_{0}$ has bounded Riemannian curvature because the Levi-Civita connection and the Chern connection only differ by the torsion (see for example [34]). In this section, we will assume $\left(M, g_{0}\right)$ to be a complete noncompact Hermitian manifold satisfying the followings.

(A) $\sup _{M}\left|R m\left(g_{0}\right)\right|+\left|T_{g_{0}}\right|^{2} \leq K_{0}$;

(B) There is an exhaustion function $\rho \geq 1$ such that

$$
\sup _{M}|\partial \rho|^{2}+|\sqrt{-1} \partial \bar{\partial} \rho| \leq K_{0}
$$

We will proceed as in [15]. Let $\kappa \in(0,1), f:[0,1) \rightarrow[0, \infty)$ be the function:

$$
f(s)= \begin{cases}0, & s \in[0,1-\kappa] \\ -\log \left[1-\left(\frac{s-1+\kappa}{\kappa}\right)^{2}\right], & s \in(1-\kappa, 1) .\end{cases}
$$

Let $\varphi \geq 0$ be a smooth function on $\mathbb{R}$ such that $\varphi(s)=0$ if $s \leq 1-\kappa+\kappa^{2}$, $\varphi(s)=1$ for $s \geq 1-\kappa+2 \kappa^{2}$

$$
\varphi(s)= \begin{cases}0, & s \in\left[0,1-\kappa+\kappa^{2}\right] \\ 1, & s \in\left(1-\kappa+2 \kappa^{2}, 1\right) .\end{cases}
$$

such that $\frac{2}{\kappa^{2}} \geq \varphi^{\prime} \geq 0$. Define

$$
\mathfrak{F}(s):=\int_{0}^{s} \varphi(\tau) f^{\prime}(\tau) d \tau .
$$

Here we collect some useful lemmas from [15].

Lemma 6.1. Suppose $0<\kappa<\frac{1}{8}$. Then the function $\mathfrak{F} \geq 0$ defined above is smooth and satisfies the following:

(i) $\mathfrak{F}(s)=0$ for $0 \leq s \leq 1-\kappa+\kappa^{2}$.

(ii) $\mathfrak{F}^{\prime} \geq 0$ and for any $k \geq 1$, $\exp (-k \mathfrak{F}) \mathfrak{F}^{(k)}$ is uniformly bounded. 
(iii) For any $1-2 \kappa<s<1$, there is $\tau>0$ with $0<s-\tau<s+\tau<1$ such that

$$
1 \leq \exp (\mathfrak{F}(s+\tau)-\mathfrak{F}(s-\tau)) \leq\left(1+c_{2} \kappa\right) ; \quad \tau \exp \left(\mathfrak{F}\left(s_{0}-\tau\right)\right) \geq c_{3} \kappa^{2}
$$

for some absolute constants $c_{2}>0, c_{3}>0$.

For any $\rho_{0}>0$, let $U_{\rho_{0}}$ be the component of $\left\{x \mid \rho(x)<\rho_{0}\right\}$ containing a fixed point $p \in M$. Hence $U_{\rho_{0}}$ will exhaust $M$ as $\rho_{0} \rightarrow \infty$. For $\rho_{0}>>1$, let $F(x)=\mathfrak{F}\left(\rho(x) / \rho_{0}\right)$. Let $h_{\rho_{0}}=e^{2 F} g_{0}$. Then $\left(U_{\rho_{0}}, h_{\rho_{0}}\right)$ is a complete Hermitian metric, see [9], and $h_{\rho_{0}}=g_{0}$ if on $\left\{\rho(x)<\left(1-\kappa+\kappa^{2}\right) \rho_{0}\right\}$.

Lemma 6.2. $\left(U_{\rho_{0}}, h_{\rho_{0}}\right)$ has bounded geometry of infinite order.

Proof. This is Lemma 4.3 in [15].

Moreover, under the assumption (A) and (B), we have

Lemma 6.3. For $\rho_{0}$ sufficiently large, we have

$$
\sup _{U_{\rho_{0}}}\left|R m\left(h_{\rho_{0}}\right)\right|+\left|T\left(h_{\rho_{0}}\right)\right|^{2} \leq 2 K_{0} .
$$

Proof. This follows directly from [15, Appendix B] and Lemma 6.1.

Now we are ready to get the short-time existence for the Hermitian Ricci flow under assumption described above which covers Theorem 1.1.

Theorem 6.1. Suppose $\left(M^{n}, g_{0}\right)$ is a complete noncompact Hermitian manifold with complex dimension $n$ so that $(\mathbf{A})$ and $(\mathbf{B})$ hold for some $K_{0}>0$. then there is a short-time solution to (3.1) with initial metric $g(0)=g_{0}$ on $M \times\left[0, c_{n} K_{0}^{-1}\right]$ which satisfies

$$
\sup _{M \times\left[0, c_{n} K_{0}^{-1}\right]}\left(|R m(g)|+\left|T_{g}\right|^{2}\right) \leq 4 K_{0} .
$$

Moreover, for all $m \in \mathbb{N}$, there is $C\left(n, m, K_{0}\right)>0$ so that on $M \times\left(0, c_{n} K_{0}^{-1}\right]$

$$
\left|\nabla^{m} T_{g}\right|^{2}+\left|\nabla^{m} R m(g)\right|^{2} \leq \frac{C\left(n, m, K_{0}\right)}{t^{m}} .
$$

Proof. Let $\left(U_{\rho_{i}}, g_{0, i}\right)$ be the sequence of Hermitian metric constructed using above method. By Lemma 6.2 and Theorem 5.1, there is a short-time solution $g_{i}(t)$ to (3.1) on each $U_{\rho_{i}}$ with initial metric $g_{0, i}=h_{\rho_{i}}$. Let $\tau_{i}$ be the maximal time such that

$$
\left(1+\epsilon_{n}\right)^{-1} g_{0, i} \leq g_{i}(t) \leq\left(1+\epsilon_{n}\right) g_{0, i} \quad \text { on } U_{\rho_{i}} \times\left[0, \tau_{i}\right]
$$

where $\epsilon_{n}$ is the constant from Proposition 4.1. By Proposition 4.1, $g_{i}(t)$ satisfies

$$
\sup _{U_{\rho_{i}} \times\left[0, \tau_{i}\right]} \max \left\{\left|R m\left(g_{i}\right)\right|,\left|T_{g_{i}}\right|^{2}\right\}<+\infty
$$

By Lemma 3.6 and (6.4), the function $F=|T|^{4}+|R m|^{2}$ is bounded and satisfies

$$
\left(\frac{\partial}{\partial t}-\Delta\right) F \leq c_{n} F^{\frac{3}{2}} .
$$


Therefore, we may apply maximum principle (see for example [12, Lemma 3.4]) to conclude that on $\left[0, \tau_{i}\right] \cap\left[0, c_{n} K_{0}^{-1}\right]$,

$$
\sup _{U_{\rho_{i}}}\left\{\left|R m\left(g_{i}\right)\right|+\left|T_{g_{i}}\right|^{2}\right\}<4 K_{0} \text {. }
$$

Claim 6.1. There is $c_{n}>0$ such that $\tau_{i} \geq c_{n} K_{0}^{-1}$ for all $i \in \mathbb{N}$.

Proof of Claim. Suppose $\tau_{i}<c_{n} K_{0}^{-1}$. Since

$$
\left(1+\epsilon_{n}\right)^{-1} g_{0, i} \leq g_{i}(t) \leq\left(1+\epsilon_{n}\right) g_{0, i}
$$

on $U_{\rho_{i}} \times\left[0, \tau_{i}\right)$. By the above discussion, if $c_{n}$ is sufficiently small, then (6.5) holds on $\left[0, \tau_{i}\right]$.

By Proposition 4.1 and Proposition 4.2, for any $m \in \mathbb{N}$, there is $C\left(n, m, U_{\rho_{i}}\right)>$ 0 such that on $U_{\rho_{i}} \times\left[0, \tau_{i}\right)$,

$$
\left|\nabla^{m} R m\left(g_{i}(t)\right)\right| \leq C\left(n, m, U_{\rho_{i}}\right) .
$$

Denote $\tilde{\nabla}=\nabla_{g_{0, i}}$. When $m=1$, since $\partial_{t}(\tilde{\nabla} g)=(\tilde{\nabla}-\nabla) S+\nabla S$

$$
\partial_{t}|\tilde{\nabla} g|^{2} \leq C_{1}+C_{1}|\tilde{\nabla} g|^{2}
$$

Hence, $\left|\nabla_{g_{0, i}} g_{i}(t)\right| \leq C\left(n, U_{\rho_{i}}\right)$ on $\left[0, \tau_{i}\right)$. Inductively, we can show that for any $m \in \mathbb{N}$, there is $C\left(n, m, U_{\rho_{i}}\right)$ such that on $U_{\rho_{i}} \times\left[0, \tau_{i}\right)$,

$$
\left|\nabla_{g_{0, i}}^{m} g_{i}(t)\right| \leq C\left(n, m, U_{\rho_{i}}\right) .
$$

Therefore, we may take subsequent limit on $g_{i}(t), t \rightarrow \tau_{i}$ to obtain $g_{i}\left(\tau_{i}\right)$ which has bounded geometry of infinity order. By Theorem 5.1, $g_{i}(t)$ exists on $\left[0, \tau_{i}+\epsilon\right)$ for some $\epsilon>0$. Moreover, if $c_{n}$ is small enough, then (6.5) implies that

$$
\left(1+\epsilon_{n}\right)^{-1} g_{0, i} \leq g_{i}(t) \leq\left(1+\epsilon_{n}\right) g_{0, i}
$$

holds on $\left[0, \tau_{i}+\epsilon\right)$ which contradicts with the maximality.

By (6.5), Proposition 4.2 and flow equation (3.1), we can use similar argument as above to show that on any compact set $\Omega$ and any $m \in \mathbb{N}$, there is $C\left(m, n, K_{0}, \Omega\right)>0$ such that for any $i>>1$, we have on

$$
\sup _{\Omega \times\left[0, c_{n} K_{0}^{-1}\right]}\left|\nabla_{g_{0}}^{m} g_{i}(t)\right| \leq C\left(m, n, K_{0}, \Omega\right) .
$$

Hence, we may take a subsequence $i_{k} \rightarrow \infty$ to obtain a limiting solution $g(t)$ on $M \times\left[0, c_{n} K_{0}^{-1}\right]$ with $g(0)=g_{0}$ and

$$
\sup _{M \times\left[0, c_{n} K_{0}^{-1}\right]}\left\{|R m(g)|+\left|T_{g}\right|^{2}\right\} \leq 4 K_{0} .
$$

The higher order derivatives follows from Proposition 4.3. 


\section{HeRmitian RiCCi FLOW ON NONPOSITIVELY CURVED MANiFOLDS}

In this section, we will apply the Hermitian flow to study complete noncompact Hermitian manifolds with non-positive bisectional curvature. In particular, we will generalize the preservation of non-positive Chern-Ricci curvature in [14] to complete noncompact case. We will first prove the following.

Theorem 7.1. Suppose $(M, g(t))$ is a complete noncompact solution to (3.1) on $M \times[0, \tau]$ with

$$
\sup _{M \times[0, \tau]}\left(|R m|+|T|^{2}\right) \leq K
$$

for some $K>1$. If $g(0)=g_{0}$ has non-positive bisectional curvature, then there is $c_{1}(n), c_{2}(n)>0$ such that for all $(x, t) \in M \times[0, \tau] \cap\left[0, c_{1} K^{-1}\right]$,

(1) $\operatorname{Ric}_{t} \leq 0$;

(2) $\left|R_{u \bar{v} x \bar{x}}\right|^{2} \leq\left(20+c_{2} \sqrt{K t}\right)\left|g_{x \bar{x}}\right|^{2}\left|R_{u \bar{u}}\right|\left|R_{v \bar{v}}\right|$ for all $x, u, v \in T^{1,0} M$.

In fact, the curvature preservation conditions were first considered in [3] where they considered Riemannian manifolds with nonnegative sectional curvature. We would like to point out that to establish weak maximum principle on curvature conditions along noncompact flow with bounded curvature, usually one will consider $R_{i \bar{j} k \bar{l}}^{\epsilon}=R_{i \bar{j} k \bar{l}}-\epsilon \rho B_{i \bar{j} k \bar{l}}$ where $\rho$ is a distance function from some fixed point (see for example [7, Chapter 12]) so that one can localize the argument on compact set. By showing that $R^{\epsilon}$ is " $\epsilon$-close" to the desired curvature conditions, one can show that $R$ satisfies the goal by letting $\epsilon \rightarrow 0$. However, since the second curvature condition in Theorem 7.1 does not explicitly satisfy the null vector condition (see for example [7, Theorem 12.33], this approach fails due to the presence of a quadratic term. We here take an alternative approach relying on parabolic rescaling argument. We first prove the following weaker version.

Proposition 7.1. Under the assumption in Theorem 7.1, there is $c_{1}(n), c_{2}(n)>$ 0 such that for all $(x, t) \in M \times[0, \tau] \cap\left[0, c_{1} K^{-1}\right]$, the curvature type tensor $\hat{R}_{i \bar{j} k \bar{l}}=R_{i \bar{j} k \bar{l}}-B_{i \bar{j} k \bar{l}}$ satisfies

(1) $\operatorname{Ric}(\hat{R}) \leq-e^{-c_{2} K t} g$;

(2) $\left|\hat{R}_{u \bar{v} x \bar{x}}\right|^{2} \leq\left(20+c_{2} \sqrt{K t}\right)\left|g_{x \bar{x}}\right|^{2}\left|\hat{R}_{u \bar{u}}\right|\left|\hat{R}_{v \bar{v}}\right|$ for all $x, u, v \in T^{1,0} M$.

We first prove Theorem 7.1 by assuming the Proposition 7.1

Proof of Theorem 7.1. For any $L>>1$, define $\tilde{g}(t)=L^{-1} g(L t)$ on $M \times$ $\left[0, \tau L^{-1}\right]$. Then $\tilde{g}(0)$ has non-positive bisectional curvature and $\tilde{g}(t)$ satisfies

$$
\sup _{M \times[0, \tau / L]}\left(|\widetilde{R m}|+|\tilde{T}|^{2}\right) \leq K L .
$$

Apply Proposition 7.1 on $\tilde{g}(t)$ and then rescale it back to $g(t)$, we have for $t \in[0, \tau] \cap\left[0, c_{1} K^{-1}\right], g(t)=L \tilde{g}(t / L)$ satisfies

(1) $\operatorname{Ric}(g(t)) \leq L^{-1}\left(n+1-e^{-c_{2} K t}\right) g(t)$; 
(2) for all $x, u, v \in T^{1,0} M$,

$$
\begin{aligned}
\left|R_{u \bar{v} x \bar{x}}-L^{-1} B_{u \bar{v} x \bar{x}}\right|^{2} \leq & \left(20+c_{2} \sqrt{K t}\right)\left|g_{x \bar{x}}\right|^{2}\left|R_{u \bar{u}}-L^{-1}(n+1) g_{u \bar{u}}\right| \\
& \times\left|R_{v \bar{v}}-L^{-1}(n+1) g_{v \bar{v}}\right| .
\end{aligned}
$$

Since this is true for all $L>>1$, the conclusion follows by letting $L \rightarrow \infty$.

Proof of Proposition 7.1. By (7.1), we may assume

$$
\frac{1}{2} g_{0} \leq g(t) \leq 2 g_{0}
$$

on $M \times[0, \tau] \cap\left[0, c_{n} K^{-1}\right]$. Let $z_{0} \in M$ and $d_{g_{0}}\left(x, z_{0}\right)$ be the distance from $z_{0}$ using the metric $g_{0}$. Let $\phi$ be a cutoff function on $[0,+\infty)$ such that $\phi \equiv 1$ on $[0,1]$, vanishes outside $[0,2]$ and satisfies

$$
\left|\phi^{\prime}\right|^{2} \leq 100 \phi, \quad \phi^{\prime \prime} \geq-100 \phi .
$$

For any $r_{0}>K^{10}$, let $\Phi(x, t)=\phi\left(\frac{d_{0}\left(x, z_{0}\right)}{r_{0}}\right)$ and define a curvature type tensor

$$
W_{i \bar{j} k \bar{l}}=\Phi R_{i \bar{j} k \bar{l}}-B_{i \bar{j} k \bar{l}} .
$$

We will use $W_{i \bar{j}}$ to denote $W_{i \bar{j} k \bar{l}} g^{k \bar{l}}$ as well.

Claim 7.1. There is $c_{1}(n), c_{2}(n)>0$ such that for all $r_{0}>>1, t \in[0, \tau] \cap$ $\left[0, c_{1} K^{-1}\right]$,

(a) $W_{i \bar{j}}<-e^{-c_{2} K t} g_{i \bar{j}}$;

(b) $\left|W_{u \bar{v} x \bar{x}}\right|^{2}<\left(20+c_{2} \sqrt{K t}\right)\left|g_{x \bar{x}}\right|^{2}\left|W_{u \bar{u}}\right|\left|W_{v \bar{v}}\right|$ for all $x, u, v \in T^{1,0} M$.

Proof of Claim. We take $c_{1}=\frac{1}{2 c_{2}}$. We will specify the choice of $c_{2}$ in the proof below. The proof is similar to [14, Lemma 4.1] except that we have to take care of the cutoff function. Clearly, the claim is true at $t=0$, see [14, Lemma 4.2] for detailed computation. Due to the cutoff function $\Phi$, if the claim is false, there is $t_{0} \in(0, \tau] \cap\left(0, c_{1} K^{-1}\right]$ such that both (a) and (b) are true on $\left[0, t_{0}\right)$ and one of them fails at $t=t_{0}$. In particular, we have for all $z \in M$, $t \in\left[0, t_{0}\right], y, u, v \in T_{z}^{1,0} M$ with $|y|=1$,

$$
\begin{aligned}
W_{y, \bar{y}} & \leq-e^{-c_{2} K t} \\
\left|W_{u \bar{v} y \bar{y}}\right|^{2} & <\left(20+c_{2} \sqrt{K t}\right) W_{u \bar{u}} W_{v \bar{v}} .
\end{aligned}
$$

As in [17, Page 1599], we may use polarization and (7.1) to infer that for any $e_{k}, e_{l} \in T^{1,0}$ with unit 1 and $e_{i}, e_{j} \in T^{1,0}$,

$$
\left|W_{i \bar{j} k \bar{l}}\right|^{2} \leq C_{n} W_{i \bar{i}} W_{j \bar{j}}, \quad\left|W_{i \bar{j} k \bar{l}}\right|^{2} \leq C_{n} K\left|W_{i \bar{i}}\right| .
$$

Case 1: Condition (a) is true on $\left[0, t_{0}\right)$ and fails at $t=t_{0}$. Then there is $p \in M, X_{0} \in T_{p}^{1,0} M$ with $\left|X_{0}\right|=1$ such that

$$
W_{X_{0}, \bar{X}_{0}}=-e^{-c_{2} K t} .
$$

Consider the following tensor

$$
A_{i \bar{j}}=\Phi R_{i \bar{j}}+\left[e^{-c_{2} K t}-(n+1)\right] g_{i \bar{j}}=W_{i \bar{j}}+e^{-c_{2} K t} g_{i \bar{j}}
$$


which satisfies $A\left(X_{0}, \bar{X}_{0}\right)=0$ and $A(Y, \bar{Y}) \leq 0$ for all $Y \in T_{x}^{1,0} M, x \in M$. We may assume $\left|X_{0}\right|_{g\left(t_{0}\right)}=1$ by rescaling.

Extend $X_{0}$ locally to a $T^{1,0}$ vector field around $\left(p, t_{0}\right)$ such that at $\left(p, t_{0}\right)$,

$$
\nabla_{\bar{q}} X^{p}=0 ; \quad \nabla_{p} X^{q}=T_{p l}^{q} X^{l}
$$

Locally, $X=X^{i} \frac{\partial}{\partial z^{i}}$. We will denote $\bar{X}=\overline{X^{i}} \frac{\partial}{\partial \bar{z}^{i}}=X^{\bar{i}} \frac{\partial}{\partial \bar{z}^{i}}$. Then $A(X, \bar{X})$ defined a function locally and satisfies

$$
\left.\square\right|_{\left(p, t_{0}\right)} A(X, \bar{X}) \geq 0
$$

where we denote $\left(\frac{\partial}{\partial t}-\Delta\right)$ by $\square$ for notational convenience. Now we compute the evolution equation for $A(X, \bar{X})$. At $\left(p, t_{0}\right)$,

$$
\begin{aligned}
\frac{\partial}{\partial t} A(X, \bar{X}) & =\left(\partial_{t} A_{i \bar{j}}\right) X^{i} X^{\bar{j}}+A_{i \bar{j}}\left(\partial_{t} X^{i} X^{\bar{j}}+X^{i} \partial_{t} X^{\bar{j}}\right) \\
& =\left[\Phi \cdot \partial_{t} R_{i \bar{j}}-\left[e^{-c_{2} K t}-(n+1)\right] S_{i \bar{j}}-c_{2} K e^{-c_{2} K t} g_{i \bar{j}}\right] X^{i} X^{\bar{j}} \\
& \leq \Phi \cdot \partial_{t} R_{i \bar{j}} \cdot X^{i} X^{\bar{j}}-\frac{1}{2} c_{2} K .
\end{aligned}
$$

provided that $c_{2}>>1$ is sufficiently large. Here we have used (17.1) and the fact that for any $Y \in T_{p}^{1,0} M$,

$$
A_{X_{0} \bar{Y}}=0
$$

Now we compute the $\Delta A(X, \bar{X})$. We may in addition assume that at $\left(p, t_{0}\right)$, $g_{i \bar{j}}=\delta_{i \bar{j}}$. Using $\nabla g=0$, (7.6) and (17.9), we have

$$
\begin{aligned}
& \Delta A(X, \bar{X}) \\
= & \frac{1}{2} g^{r \bar{s}}\left(\nabla_{r} \nabla_{\bar{s}}+\nabla_{\bar{s}} \nabla_{r}\right)\left(A_{i \bar{j}} X^{i} X^{\bar{j}}\right) \\
= & \Delta\left(\Phi R_{i \bar{j}}\right) \cdot X^{i} X^{\bar{j}}+A_{i \bar{j} ; \bar{r}} T_{r p}^{i} X^{p} X^{\bar{j}}+A_{i \bar{j} ; r} T_{\bar{r} \bar{q}}^{\bar{j}} X^{i} X^{\bar{q}}+A_{i \bar{j}} T_{r p}^{i} T_{\bar{r} \bar{q}}^{\bar{j}} X^{p} X^{\bar{q}} \\
= & \Delta \Phi \cdot R_{i \bar{j}} X^{i} X^{\bar{j}}+\Phi \cdot \Delta R_{i \bar{j}} X^{i} X^{\bar{j}}+2 \operatorname{Re}\left(\nabla_{r} \Phi \cdot \nabla_{\bar{r}} R_{i \bar{j}} \cdot X^{i} X^{\bar{j}}\right) \\
& +\nabla_{\bar{r}}\left(\Phi R_{i \bar{j}}\right) T_{r p}^{i} X^{p} X^{\bar{j}}+\nabla_{r}\left(\Phi R_{i \bar{j}}\right) T_{\bar{r} \bar{q}}^{\bar{j}} X^{i} X^{\bar{q}}+A_{i \bar{j}} T_{r p}^{i} T_{\bar{r} \bar{q}}^{\bar{j}} X^{p} X^{\bar{q}} \\
= & \Delta \Phi \cdot R_{i \bar{j}} X^{i} X^{\bar{j}}+\Phi \cdot \Delta R_{i \bar{j}} X^{i} X^{\bar{j}}+2 \operatorname{Re}\left(\nabla_{r} \Phi \cdot \nabla_{\bar{r}}\left(R_{X \bar{X}}\right)\right) \\
& +\Phi R_{i \bar{j} ; \bar{r}} T_{r p}^{i} X^{p} X^{\bar{j}}+\Phi R_{i \bar{j} ; r} T_{\bar{r} \bar{q}}^{\bar{j}} X^{i} X^{\bar{q}}+A_{i \bar{j}} T_{r p}^{i} T_{\bar{r} \bar{q}}^{\bar{j}} X^{p} X^{\bar{q}} .
\end{aligned}
$$


By combining (7.8) and (7.10), we have

$$
\begin{aligned}
\square A(X, \bar{X}) \leq & -\frac{1}{2} c_{2} K+\Phi \square R_{i \bar{j}} \cdot X^{i} X^{\bar{j}}-2 \operatorname{Re}\left(\nabla_{r} \Phi \cdot \nabla_{\bar{r}}\left(R_{X \bar{X}}\right)\right) \\
& -\Phi R_{i \bar{j} ; \bar{r}} T_{r p}^{i} X^{p} X^{\bar{j}}-\Phi R_{i \bar{j} ; r} T_{\bar{r} \bar{q}}^{\bar{j}} X^{i} X^{\bar{q}} \\
& -\Delta \Phi \cdot R_{i \bar{j}} X^{i} X^{\bar{j}}-A_{i \bar{j}} T_{r p}^{i} T_{\bar{r} \bar{q}}^{\bar{j}} X^{p} X^{\bar{q}} \\
= & -\frac{1}{2} c_{2} K-\Delta \Phi \cdot R_{X \bar{X}}+\left(n+1-e^{-c_{2} K t}\right)\left|T_{r X}^{i}\right|^{2} \\
& -2 \operatorname{Re}\left(\nabla_{r} \Phi \cdot \nabla_{\bar{r}}\left(R_{X \bar{X}}\right)\right)+\Phi R_{X \bar{X} p}{ }^{q} R_{q}{ }^{p} \\
& -\left(n+1-e^{-c_{2} K t}\right) S_{X \bar{X}}
\end{aligned}
$$

where we have used (7.9) in the last step.

Since at $\left(p, t_{0}\right)$, we have $\nabla_{r} A_{X \bar{X}}=0$. Hence,

$$
\nabla_{\bar{r}} \Phi \cdot R_{X \bar{X}}+\Phi \nabla_{\bar{r}} R_{X \bar{X}}=\left((n+1)-e^{-c_{2} K t}\right) g_{X \bar{j}} T_{\bar{r} \bar{X}}^{\bar{j}}
$$

On the other hand, as $\Phi R_{X \bar{X}}=\left(n+1-e^{-c_{2} K t}\right)$ at $\left(p, t_{0}\right)$ and $t_{0} \in[0, \tau] \cap$ $\left[0, c_{1} K^{-1}\right]$,

$$
\Phi \geq \frac{1}{(n+1) K}\left[n+1-e^{-c_{2} K t}\right] \geq \frac{n}{(n+1) K} .
$$

Hence, by using the properties of $\phi$ and combining with (7.1), (7.3) and $r_{0}>K^{2}$, we have

$$
\begin{aligned}
-2 \operatorname{Re}\left(\nabla_{r} \Phi \cdot \nabla_{\bar{r}}\left(R_{X \bar{X}}\right)\right) & =\frac{\left|\nabla_{r} \Phi\right|^{2}}{\Phi} R_{X \bar{X}}-\frac{n+1-e^{-c_{2} K t}}{\Phi} g_{X \bar{j}} T_{\bar{r} \bar{X}}^{\bar{j}} \nabla_{r} \Phi \\
& \leq \frac{c_{n}^{\prime}}{\sqrt{r_{0}}}
\end{aligned}
$$

Similarly,

$$
-\Delta \Phi \cdot R_{X \bar{X}} \leq \frac{c_{n}}{\sqrt{r_{0}}} .
$$

We now combine (7.11), (7.12), (7.13) and (7.1) to show that if $c_{2}$ is sufficiently large depending only on $n$, then

$$
\begin{aligned}
\square A(X, \bar{X}) & \leq-\frac{1}{3} c_{2} K+W_{X \bar{X} p \bar{q}} R_{q \bar{p}} \\
& \leq-\frac{1}{3} c_{2} K+C_{n} K\left|W_{X \bar{X}}\right| \\
& <0
\end{aligned}
$$

where we have used (77.5). But this contradicts with (7.7).

Case 2: Condition (b) is fail at $t=t_{0}$. Then there is $p \in M, x_{0}, u_{0}, v_{0} \in$ $T_{p}^{1,0} M$ with $\left|x_{0}\right|_{t_{0}}=1$ such that

$$
\left|W_{u_{0} \bar{v}_{0} x_{0} \bar{x}_{0}}\right|^{2}=\left(20+c_{2} \sqrt{K t}\right) W_{u_{0} \bar{u}_{0}} W_{v_{0} \bar{v}_{0}}
$$


By rescaling, we may assume $\left|u_{0}\right|_{t_{0}}=\left|v_{0}\right|_{t_{0}}=1$. As in case 1 , we extend $x_{0}, u_{0}, v_{0}$ to local vector field $X, U, V$ around $\left(p, t_{0}\right)$. We extend $x_{0}$ so that along each geodesics $\gamma$ emanating from $p, \nabla_{\dot{\gamma}} X=0$ at $t=t_{0}$ and constant in $t$. On the other hand, we extend $u_{0}, v_{0}$ to $U$ and $V$ such that at $\left(p, t_{0}\right)$,

$$
\begin{array}{rlrlrl}
\nabla_{\bar{s}} U^{r} & =0, & \nabla_{p} U^{r} & =T_{p q}^{r} U^{q}, & \square U^{r} & =\frac{1}{2} S_{p}^{r} U^{p} ; \\
\nabla_{\bar{s}} V^{r} & =0, & \nabla_{p} V^{r}=T_{p q}^{r} V^{q} ; & \square V^{r} & =\frac{1}{2} S_{p}^{r} V^{p} ; \\
\nabla_{\bar{s}} X^{r} & =0, & \nabla_{p} X^{r} & =0 ; & \square X^{r} & =0 .
\end{array}
$$

Hence the function

$$
F(x, t)=g_{X \bar{X}}^{-2}\left|W_{U \bar{V} X \bar{X}}\right|^{2}-\left(20+c_{2} \sqrt{K t}\right) W_{U \bar{U}} W_{V \bar{V}}
$$

attains its local maximum at $\left(p, t_{0}\right)$ and therefore satisfies

$$
\left.\square F\right|_{\left(p, t_{0}\right)} \geq 0 .
$$

We now differentiate each of them carefully. Using (7.15) and Lemma 3.5, a similar calculation as in Case 1 yields

$$
\begin{aligned}
\square W_{U \bar{U}}= & \square W_{i \bar{j}} \cdot U^{i} U^{\bar{j}}+W_{i \bar{j}} U^{i} \square U^{\bar{j}}+W_{i \bar{j}} \square U^{i} \cdot U^{\bar{j}} \\
& -g^{r \bar{s}} W_{i \bar{j}} U_{; r}^{i} U_{; \bar{s}}^{\bar{j}}-g^{r \bar{s}} W_{i \bar{j} ; r} U^{i} U_{; \bar{s}}^{\bar{j}}-g^{r \bar{s}} W_{i \bar{j} ; \bar{s}} U_{; r}^{i} U^{\bar{j}} \\
= & \square \Phi \cdot R_{U \bar{U}}-2 \operatorname{Re}\left(g^{r \bar{s}} \Phi_{r} \nabla_{\bar{s}} R_{i \bar{j}} \cdot U^{i} U^{\bar{j}}\right)+(n+1) S_{U \bar{U}} \\
& -(n+1) \Phi S_{U \bar{U}}+(n+1) g_{i \bar{j}} g^{r \bar{s}} T_{r U}^{i} T_{\bar{s} \bar{U}}^{\bar{j}} \\
& -g^{r \bar{s}} \Phi_{r} R_{U \bar{j}} T_{\bar{s} \bar{U}}^{\bar{j}}-g^{r \bar{s}} \Phi_{\bar{s}} R_{i \bar{U}} T_{r U}^{i}+\Phi R_{U \bar{U} k}^{p} R_{p}^{k} .
\end{aligned}
$$

By Proposition 4.3, we have $|\nabla R m| \leq C(n, K) t^{-1 / 2}$. Using the choice of $\phi$, Lemma 4.1, (17.1), and $r_{0}>>K$, we have

$$
\begin{aligned}
\square W_{U \bar{U}} & \leq C_{n} K+\frac{C_{n}}{\sqrt{t}}+\Phi R_{U \bar{U} k}^{p} R_{p}^{k} \\
& \leq \frac{C_{n}}{\sqrt{t}}-C_{n} K W_{U \bar{U}}
\end{aligned}
$$

where we have used (7.4) and (7.5) in the last inequality. Similarly,

$$
\square W_{V \bar{V}} \leq \frac{C_{n}}{\sqrt{t}}-C_{n} K W_{V \bar{V}}
$$

By combining (7.17), (7.18) with (7.4), (77.5), (7.1) and using the fact that $c_{2}>>1$, we arrive at the following inequality.

$$
\begin{aligned}
& \square\left[\left(20+c_{2} \sqrt{K t}\right) W_{U \bar{U}} W_{V \bar{V}}\right] \\
\geq & -2\left(20+c_{2} \sqrt{K t}\right) \operatorname{Re}\left(g^{r \bar{s}} \nabla_{r} W_{U \bar{U}} \cdot \nabla_{\bar{s}} W_{V \bar{V}}\right)+\frac{c_{2}}{2} \sqrt{\frac{K}{t}} W_{U \bar{U}} W_{V \bar{V}} .
\end{aligned}
$$


Now we derive the evolution equation of $\left|W_{U \bar{V} X \bar{X}}\right|^{2}$. Similar to the computation of $\square W_{U \bar{U}}$, using (7.15) and Lemma 3.7, we have

$$
\begin{aligned}
& \square W_{U \bar{V} X \bar{X}}=\square(\Phi R)_{i \bar{j} k \bar{l}} \cdot U^{i} V^{\bar{j}} X^{k} X^{\bar{l}}-\square B_{i \bar{j} k \bar{l}} \cdot U^{i} V^{\bar{j}} X^{k} X^{\bar{l}} \\
& +W_{i \bar{j} k \bar{l}}\left(\square U^{i}\right) V^{\bar{j}} X^{k} X^{\bar{l}}+W_{i \bar{j} k \bar{l}} U^{i}\left(\square V^{\bar{j}}\right) X^{k} X^{\bar{l}} \\
& -g^{r \bar{s}}\left(\Phi R_{i \bar{j} k \bar{l}}\right)_{; r} U^{i} V_{; \bar{s}}^{\bar{j}} X^{k} X^{\bar{l}}-g^{r \bar{s}}\left(\Phi R_{i \bar{j} k \bar{l}}\right)_{; \bar{s}} U_{; r}^{i} V^{\bar{j}} X^{k} X^{\bar{l}} \\
& -W_{i \bar{j} k \bar{l}} U_{; r}^{i} V_{; \bar{s}}^{\bar{j}} X^{k} X^{\bar{l}} \\
& =\Phi g^{r \bar{s}}\left[R_{U \bar{V} r}{ }^{p} R_{p \bar{s} X \bar{X}}+R_{r \bar{V} X}{ }^{p} R_{U \bar{s} p \bar{X}}-R_{r \bar{V} p \bar{X}} R_{U \bar{s} X}{ }^{p}\right] \\
& -\frac{1}{2} \Phi\left[S_{X}^{p} R_{U \bar{V} p \bar{X}}+S_{\bar{X}}^{\bar{q}} R_{U \bar{V} X \bar{q}}\right] \\
& +\left(S_{U \bar{V}} g_{X \bar{X}}+g_{U \bar{V}} S_{X \bar{X}}+S_{U \bar{V}} g_{X \bar{X}}+g_{U \bar{V}} S_{X \bar{X}}\right) \\
& +g^{r \bar{s}} B_{i \bar{j} X \bar{X}} T_{r U}^{i} T_{\bar{s} \bar{V}}^{\bar{j}}-\frac{1}{2}\left(B_{i \bar{V} X \bar{X}} S_{U}^{i}+B_{U \bar{j} X \bar{X}} S_{\bar{V}}^{\bar{j}}\right) \\
& -\left(g^{r \bar{s}} \Phi_{r} \nabla_{\bar{s}} R_{i \bar{j} k \bar{l}} U^{i} V^{\bar{j}} X^{k} X^{\bar{l}}+g^{r \bar{s}} \Phi_{\bar{s}} \nabla_{r} R_{i \bar{j} k l} U^{i} V^{\bar{j}} X^{k} X^{\bar{l}}\right) \\
& -\left(g^{r \bar{s}} \Phi_{r} T_{\bar{s} \bar{V}}^{\bar{j}} R_{U \bar{j} X \bar{X}}+g^{r \bar{s}} \Phi_{\bar{s}} T_{r U}^{i} R_{i \bar{V} X \bar{X}}\right)+\square \Phi \cdot R_{U \bar{V} X \bar{X}} \cdot
\end{aligned}
$$

The equation of $\square W_{V \bar{U} X \bar{X}}$ is similar.

Noted that we have $|\nabla R m| \leq C(n, K) t^{-1 / 2}$ by Proposition 4.3. Therefore by combining (7.20), (7.1), (7.5) and (17.4) and using the property of $\phi$ and $r>>K$, we have

$$
\begin{aligned}
& \square\left|W_{U \bar{V} X \bar{X}}\right|^{2} \leq-\left|\nabla W_{U \bar{V} X \bar{X}}\right|^{2}-\left|\bar{\nabla} W_{U \bar{V} X \bar{X}}\right|^{2}+C_{n} \sqrt{\frac{K}{t}} W_{U \bar{U}} W_{V \bar{V}} \\
& +\Phi\left|R_{\bar{V} X}^{\bar{s}}{ }^{p} R_{U \bar{s} p \bar{X}}-R_{\bar{V} p \bar{X}}^{\bar{s}} R_{U \bar{s} X}^{p}\right|\left|W_{V \bar{U} X \bar{X}}\right| \\
& +\Phi\left|R_{\bar{U} X}^{\bar{s}}{ }^{p} R_{V \bar{s} p \bar{X}}-R_{\bar{U} p \bar{X}}^{\bar{s}} R_{V \bar{s} X}{ }^{p}\right|\left|W_{U \bar{V} X \bar{X}}\right|
\end{aligned}
$$

The main trouble is the quadratic term appeared on the right hand side because of $\Phi$ there and $U, V$ take places at different curvature term.

$$
\begin{aligned}
& \Phi\left|R_{\bar{V} X}^{\bar{s}}{ }^{p} R_{U \bar{s} p \bar{X}}-R_{\bar{s} p \bar{X}}^{\bar{s}} R_{U \bar{s} X}{ }^{p}\right| \\
\leq & \frac{1}{\Phi}\left|W^{\bar{s}}{ }_{\bar{V} X}^{p} W_{U \bar{s} p \bar{X}}-W^{\bar{s}}{ }_{\bar{V} p \bar{X}} W_{U \bar{s} X}{ }^{p}\right|+C_{n} K \\
\leq & \left(\frac{C_{n}}{\Phi}+C_{n} K\right) \sqrt{W_{U \bar{U}} W_{V \bar{V}}}
\end{aligned}
$$


where we have used (7.4) and (7.5) in the last inequality. On the other hand, since at $\left(p, t_{0}\right)$,

$$
\begin{aligned}
10 & \leq\left(20+c_{2} \sqrt{K t}\right) W_{U \bar{U}} W_{V \bar{V}} \\
& =\left|\Phi R_{U \bar{V} X \bar{X}}-B_{U \bar{V} X \bar{X}}\right|^{2} \\
& \leq 8+2 \Phi^{2} K^{2} .
\end{aligned}
$$

Therefore, $\Phi \geq K^{-1}$ at $\left(p, t_{0}\right)$. Combines with (7.23) ,

$$
\Phi\left|R_{\bar{V} X}^{\bar{s}} R_{U \bar{s} p \bar{X}}-R_{\bar{V} p \bar{X}}^{\bar{s}} R_{U \bar{s} X}^{p}\right| \leq C_{n} K \sqrt{W_{U \bar{U}} W_{V \bar{V}}} .
$$

And hence at $\left(p, t_{0}\right)$,

$$
\begin{aligned}
\left(\frac{\partial}{\partial t}-\Delta\right) F \leq & 2\left(20+c_{2} \sqrt{K t}\right) \mathbf{R e}\left(g^{r \bar{s}} \nabla_{r} W_{U \bar{U}} \cdot \nabla_{\bar{s}} W_{V \bar{V}}\right) \\
& -\left|\nabla W_{U \bar{V} X \bar{X}}\right|^{2}-\left|\bar{\nabla} W_{U \bar{V} X \bar{X}}\right|^{2} \\
& -\frac{c_{2}}{4} \sqrt{\frac{K}{t}} W_{U \bar{U}} W_{V \bar{V}}+2 S_{X \bar{X}}\left|W_{U \bar{V} X \bar{X}}\right|^{2}
\end{aligned}
$$

By using the fact that $\nabla F=0$ and $F=0$ at $\left(p, t_{0}\right)$, one can conclude that

$$
2\left(20+c_{2} \sqrt{K t}\right) \operatorname{Re}\left(g^{r \bar{s}} \nabla_{r} W_{U \bar{U}} \cdot \nabla_{\bar{s}} W_{V \bar{V}}\right) \leq\left|\nabla W_{U \bar{V} X \bar{X}}\right|^{2}+\left|\bar{\nabla} W_{U \bar{V} X \bar{X}}\right|^{2} .
$$

Using (7.1) and (7.5) again, we deduce that

$$
2 S_{X \bar{X}}\left|W_{U \bar{V} X \bar{X}}\right|^{2} \leq C_{n} K W_{U \bar{U}} W_{V \bar{V}}
$$

and hence at $\left(p, t_{0}\right)$,

$$
\left(\frac{\partial}{\partial t}-\Delta\right) F<-\frac{c_{2}}{8} \sqrt{\frac{K}{t}} W_{U \bar{U}} W_{V \bar{V}}
$$

which contradicts with (7.16) provided that $c_{2}(n)>>1$. This proves the claim.

The assertion follows by letting $r_{0} \rightarrow \infty$.

An immediate consequence is the following splitting theorem in Kähler case based on the strong maximum principle along the noncompact Kähler-Ricci flow and the De Rham decomposition theorem.

Corollary 7.1. Let $g(t)$ be a complete solution to the Kähler-Ricci flow on a noncompact simply connected complex manifold $M^{n}$ with bounded curvature. If the initial metric $g_{0}$ has non-positive bisectional curvature. Then for sufficiently small $t>0$, either $g(t)$ has negative Ricci curvature on $M$ or $(M, g(t))$ splits holomorphically isometrically into a product $\mathbb{C}^{k} \times N^{n-k}$.

Proof. Since we have established the preservation of curvature condition in Theorem 7.1 in the noncompact case. By theorem 7.1, the tensor $e^{-A t} R i c_{g(t)}$ satisfies the null vector condition for $A$ sufficiently large and $t$ sufficiently small. Then we can apply the standard Strong maximum principle argument in [7, 
Theorem 12.50] to show that the kernel of $R i c_{g(t)}$ is parallel in space and time, see also [17, Page 1602-1603] for the original argument in compact case. The flatness of the kernel follows by the second conclusion in Theorem 7.1 ,

As an application of Strong maximum principle, we have the following.

Theorem 7.2. Suppose $(M, g(t)), t \in[0, \tau]$ is a complete solution to (3.1) satisfying the assumption in Theorem 7.1. If the initial metric $g_{0}$ has quasinegative Chern-Ricci curvature, then Ric $(g(t))<0$ on $M \times\left(0, \tau^{\prime}\right]$ for some $\tau^{\prime}>0$. In particular, $M$ supports a Kähler metric which is possibly incomplete.

Proof. The argument is exactly the same as the argument in the compact case [14] except that we construct the barrier by solving Dirichlet problem on some compact set instead of the whole manifold $M$. We will closely follow the argument in [7]. We here only point out the necessary modifications. Let $\tau^{\prime}=\min \left\{c_{1} K^{-1}, \tau\right\}$ be the number obtained from Theorem 7.1 .

Let $y \in M$ be a point at which the Chern-Ricci curvature of $g_{0}$ is negative. For any $x \in M$, let $\Omega$ be a connected open set with smooth boundary and containing both $x$ and $y$. Let $\phi_{0}$ be a smooth nonnegative function such that $\phi_{0}(y)>0, \phi_{0}=0$ near $\partial \Omega$ and

$$
\operatorname{Ric}\left(g_{0}\right)+\phi_{0} g_{0} \leq 0
$$

on $\Omega$. Let $\phi(z, t)$ be the solution to the heat equation

$$
\begin{gathered}
\left(\frac{\partial}{\partial t}-\Delta_{g(t)}\right) \phi(x, t)=0, \quad \text { on } \Omega \times\left[0, \tau^{\prime}\right] ; \\
\phi(x, 0)=\phi_{0}, \quad \text { and }\left.\quad \phi(x, t)\right|_{\partial \Omega}=0 .
\end{gathered}
$$

It then follows by strong maximum principle that $\phi(x, t)>0$ on $\Omega \times\left(0, \tau^{\prime}\right]$. We may assume that $\phi(x, t) \leq 1$ by rescaling. As in [14], we consider the tensor

$$
A^{\epsilon}=R i c_{g(t)}+e^{-k t} \phi^{2} g(t)-\epsilon e^{B t} g(t)
$$

where $B, k$ is some large constant. Then if $A^{\epsilon}$ fail to be negative on $\bar{\Omega} \times\left[0, \tau^{\prime}\right]$, it can only happen at $\left(x_{0}, t_{0}\right)$ where $x_{0} \in \operatorname{int}(\Omega)$ and $t_{0}>0$. Without loss of generality, we may assume $t_{0}$ to be the first time such that $A^{\epsilon}$ fails to be negative. And we may apply second derivatives test at $t=t_{0}$.

Now the argument in [14] can be carried over since the argument is purely local. Hence we can show that for any $\epsilon>0, A^{\epsilon}<0$ on $\Omega \times\left[0, \tau^{\prime}\right]$. By letting $\epsilon \rightarrow 0$, it shows that $\operatorname{Ric}(x, t)<0$ for $t \in\left(0, \tau^{\prime}\right]$. Since $x$ is arbitrary, this completes the proof. By taking $h=-\operatorname{Ric}\left(g\left(t_{1}\right)\right)$, we see that $(M, h)$ is a Kähler manifold since the Chern-Ricci form is $d$-closed by definition.

\section{Existence OF KäHLER-Einstein METRIC}

In this section, we will show that under the assumption in Theorem [7.1, if in addition $g_{0}$ has uniformly negative Chern-Ricci curvature outside a compact set, then $M$ supports a complete Kähler-Einstein metric with negative scalar curvature. 
We first show that the uniform negativity at infinity will be preserved along the flow with bounded Chern curvature and torsion.

Proposition 8.1. Suppose $(M, g(t)), t \in[0, \tau]$ is a complete solution to (3.1) with

$$
\sup _{M \times[0, \tau]}|R m|+|T|^{2} \leq K_{0}
$$

for some $K_{0}>0$. If there is $p \in M, r>0$ such that outside $B_{g_{0}}(p, r)$, the Chern-Ricci curvature Ric $\left(g_{0}\right)<-\delta$ for some $\delta>0$, then there is $\tilde{\tau}\left(n, K_{0}, \delta\right)>$ 0 so that on $[0, \tau] \cap[0, \tilde{\tau}]$, Ric $(g(t))<-\delta / 2$ outside $B_{g_{0}}(p, r+1)$.

Proof. The proof is standard. For the sake of completeness, we give the proof here. By rescaling, we may assume $\delta=1$. Let $z \in M$ such that $B_{g_{0}}(z, 1)$ is disjoint from $B_{g_{0}}(p, r)$. Let $\phi$ be a cutoff function on $[0,+\infty)$ such that $\phi \equiv 1$ on $[0,1 / 2]$, vanishes outside $[0,1]$ and satisfies

$$
\left|\phi^{\prime}\right|^{2} \leq 100 \phi, \quad \phi^{\prime \prime} \geq-100 \phi .
$$

Let $\Phi=\phi\left(d_{g_{0}}(x, z)\right)$ be a cutoff function on $M$. For $\epsilon>0$, consider the $(1,1)$ type tensor $A_{i \bar{j}}^{\epsilon}=\Phi\left(R_{i \bar{j}}+g_{i \bar{j}}\right)-(\epsilon+2 L \sqrt{t}) g_{i \bar{j}}$ with $L>>1$. Our goal is to show that for any $\epsilon>0, A^{\epsilon}<0$ for $t$ sufficiently small independent of $\epsilon$. We will omit the index $\epsilon$ for notational convenient. Clearly by continuity, it holds for $t<\tau(\epsilon, g(t))$ sufficiently small. Let $t_{0}>0$ be the first such that $A$ fails to be negative. Then at $t=t_{0}$, there is $x \in B_{g_{0}}(z, 1), X_{0} \in T_{x}^{1,0} M$ such that

$$
A_{X_{0} \bar{X}_{0}}=0 \text {. }
$$

We may assume $\left|X_{0}\right|=1$ by rescaling. Extends $X_{0}$ to local vector field $X \in$ $T^{1,0} M$ such that $\nabla X=0$ at $\left(p, t_{0}\right)$. Using the fact that $A_{X_{0} Y}=0$ for all $Y \in T^{1,0} M$ and the extension,

$$
\begin{aligned}
0 & \leq\left(\frac{\partial}{\partial t}-\Delta\right) A_{X \bar{X}} \\
& =\square A_{i \bar{j}} \cdot X^{i} X^{\bar{j}} \\
& =\square\left(\Phi R_{i \bar{j}}+\Phi g_{i \bar{j}}-\epsilon g_{i \bar{j}}-2 L \sqrt{t} g_{i \bar{j}}\right) \cdot X^{i} X^{\bar{j}} .
\end{aligned}
$$

Using the estimate on the cutoff function, Lemma 4.1, curvature assumptions, Proposition 4.3 and Lemma 3.5, we have

$$
\left(\frac{\partial}{\partial t}-\Delta\right) A_{X \bar{X}} \leq c\left(n, K_{0}\right) t^{-1 / 2}-L t^{-1 / 2}
$$

Hence if $L>>1$ is large enough, we have got contradiction. By letting $\epsilon \rightarrow 0$, we have shown that there is $L\left(n, K_{0}\right)>0$ such that for any $t \in[0, \tau]$, $x$ outside $B_{g_{0}}(p, r+1)$,

$$
R_{i \bar{j}} \leq(-1+L \sqrt{t}) g_{i \bar{j}}
$$

The assertion follows by choosing $\tilde{\tau}$ small enough.

Now we are ready to prove the existence of Kähler-Einstein metric. 
Theorem 8.1. Suppose $\left(M, g_{0}\right)$ is a complete noncompact Hermitian manifold with

$$
\sup _{M}\left\{\left|R m\left(g_{0}\right)\right|+\left|T_{g_{0}}\right|^{2}+\left|R m^{L}\left(g_{0}\right)\right|\right\}<+\infty
$$

and non-positive bisectional curvature. If in addition there is a compact set $\Omega$, $\delta>0$ such that outside $\Omega, \operatorname{Ric}\left(g_{0}\right)<-\delta g_{0}$. Then there is a Kähler-Einstein metric $g_{K E}=-\operatorname{Ric}\left(g_{K E}\right)$ on $M$. Furthermore, the curvature tensor Rm of $g_{K E}$ and all its covariant derivatives are bounded.

Proof. By Theorem 6.1, there is a short-time solution $g(t)$ starting from $g(0)=$ $g_{0}$ on $M \times[0, \tau]$ with bounded Chern curvature and torsion. By Theorem 7.2 and Proposition 8.1, there is another Hermitian metric $g(\tau)$ with

$$
-C g(\tau) \leq \operatorname{Ric}(g(\tau))<-\sigma g(\tau)
$$

for some $\sigma, C>0$. Let $h=-\operatorname{Ric}(g(\tau))$. As the Chern-Ricci curvature is $d$ closed, $h$ is a complete Kähler metric uniformly equivalent to $g(\tau)$. Moreover, the higher order estimate in Theorem 6.1 implies that for all $m \in \mathbb{N}$, there is $C\left(n, m, K_{0}\right)>0$ such that

$$
\left|\nabla_{g(\tau)}^{m} h\right| \leq C\left(n, m, K_{0}\right)
$$

Rewrite

$$
-\operatorname{Ric}(h)=-\operatorname{Ric}(g(\tau))+\sqrt{-1} \partial \bar{\partial} \log \frac{\operatorname{det} h}{\operatorname{det} g(\tau)}=h+\sqrt{-1} \partial \bar{\partial} F .
$$

By (8.2), all the covarient derivatives of $F$ with respect to $h$ are bounded. By [19, Theorem 5.1], there is a complete Kähler-Einstein metric $g_{K E}=$ $-\operatorname{Ric}\left(g_{K E}\right)$ on $M$, see also [5, 11]. Furthermore, by Shi's estimate [24] or Proposition 4.3, the curvature tensor of $g_{K E}$ and all its covariant derivatives are bounded.

\section{REFERENCES}

[1] Aubin, T., Equations du type Monge-Ampère sur les variété s kählèriennes compactes (French),Bull. Sci. Math. (2) 102 (1978), no. 1, 6395

[2] Bamler, R.,Stability of Einstein Metrics of Negative Curvature, dissertation, Princeton University, (2011)

[3] C. Böhm and B. Wilking,Nonnegative curved manifolds with finite fundamental groups admit metrics with positive Ricci curvature. Geometric and Functional Analysis 17 (2007), 665681.

[4] Cao, H.-D., Deformation of Kähler metrics to Kähler-Einstein metrics on compact Kähler manifolds, Invent. Math. 81 (1985), no. 2, 359-372.

[5] Chau, A.; Lee, M.-C., The Kähler Ricci flow around complete bounded curvature Kähler metrics, arXiv:1904.02233, accepted by Trans. Amer. Math. Soc.

[6] Cheng, S.-Y.; Yau. S.-Y., On the existence of a complete Kähler metric on noncompact complex manifolds and the regularity of Feffermans equation. Comm. Pure Appl. Math., 33(4):507544, 1980. 
[7] Chow, B.; Chu, S.-C.; Glikenstein, D.; Guether, C.; Isenberg, J.; Ivey, T.; Knopf, D.; Lu, P.; Luo, F.; Ni, L., The Ricci Flow: Techniques and Applications, Part II: Analytic Aspects, Mathematical Surveys and Monographs, vol. 144, AMS, Providence, RI, 2008.

[8] Gill, M., Convergence of the parabolic complex Monge-Ampère equation on compact Hermitian manifolds, Comm. Anal. Geom. 19 (2011), no. 2, 277303, MR 2835881, Zbl 1251.32035 .

[9] Hochard, R., Short-time existence of the Ricci flow on complete, non-collapsed 3manifolds with Ricci curvature bounded from below, arXiv:1603.08726 (2016).

[10] Huang, S.-C.; Tam, L.-F., Kähler-Ricci flow with unbounded curvature, Amer. J. Math. 140 (2018), no. 1, 189220, MR3749193.

[11] Huang, S.;, Lee, M.-C.; Tam, L.-F., Instantaneously complete Chern-Ricci flow and Kähler Einstein metrics, Calc. Var. Partial Differential Equations 58 (2019), no. 5, Art. 161, 34 pp.

[12] Huang, S.;, Lee, M.-C.; Tam, L.-F.; Tong, F., Longtime existence of Kähler Ricci flow and holomorphic sectional curvature. arXiv:1805.12328,

[13] Ladyžhenskaia O.-A.; Solonnikov, V.-A.; Uralceva, N.-N., Linear and quasi-linear equations of parabolic type, (Amer Mathematical Society, 1968).

[14] Lee, M.-C., Compact Hermitian manifolds with quasi-negative curvature, arXiv:1810.07325v4.

[15] Lee, M.-C.; Tam, L.-F., Chern-Ricci flows on noncompact manifolds. arXiv:1708.00141, to appear in JDG.

[16] Li, P.; Wang, J., Comparison theorem for Kähler manifolds and positivity of spectrum, J. Diff. Geom. 69 (2005), 43-74.

[17] Liu, G., Compact Kähler manifolds with nonpositive bisectional curvature, Geom.Func.Anal.,24(2014),1591-1607.

[18] Liu, K.-F.; Yang, X.-K., Geometry of Hermitian manifolds, Internat. J. Math. 23 (2012), no. 6, 1250055, 40 pp.

[19] Lott, J.; Zhang, Z., Ricci flow on quasi-projective manifolds, Duke Math. J. 156 (2011), no. 1, 87-123, MR2746389, Zbl 1248.53050.

[20] Mori, S., Projective manifolds with ample tangent bundles, Ann. of Math. (2), 110 (1979), pp. 593606.

[21] Sesum, N. Curvature tensor under the Ricci flow Amer. J. Math. 127 (2005), no. 6, $1315-1324$.

[22] Schnürer, O.; Schulze, F.; Simon, M., Stability of Euclidean space under Ricci flow, Communications in Analysis and Geometry 16, no. 1 (2008): 127-158.

[23] Sherman, M.; Weinkove, B., Local Calabi and curvature estimates for the Chern-Ricci flow, New York J. Math. 19 (2013), 565582, MR3119098, Zbl 1281.53069.

[24] Shi, W.-X., Deforming the metric on complete Riemannian manifolds, J. Differential Geom. 30 (1989), no. 1, 223301

[25] Shi, W.-X., Ricci Flow and the uniformization on complete noncompact Kähler manifolds, J. of Differential Geometry 45 (1997), 94220

[26] Streets, J.; Tian, G., Hermitian curvature flow. Journal of the European Mathematical Society 13.3 (2011): 601-634.

[27] Streets, J., and Tian, G., A parabolic flow of pluriclosed metrics. Int. Math. Res. Not. IMRN, 16 (2010), 31013133.

[28] Streets, J.; Tian, G.; Regularity results for the pluriclosed flow, Geom. \& Top. 17 (2013) 2389-2429.

[29] Tam, L.-F., Exhaustion functions on complete manifolds, Recent advances in geometric anal- ysis, 211215, Adv. Lect. Math. (ALM), 11, Int. Press, Somerville, MA, 2010. MR2648946, Zbl 1198.53040. 
[30] Ustinovskiy, Y., The Hermitian curvature flow on manifolds with non-negative Griffiths curvature, arXiv:1604.04813v2.

[31] Wu. D., Kähler-Einstein metrics of negative Ricci curvature on general quasiprojective manifolds. Comm. Anal. Geom., 16(2):395435, 2008.

[32] Wu, D.-M.; Yau, S.-T., Invariant metrics on negatively pinched complete Kähler manifolds, arXiv:1711.09475.

[33] Xu, G., Short-time existence of the Ricci flow on noncompact Riemannian manifolds, Trans. Amer. Math. Soc. 365 (2013), no. 11, 56055654.

[34] Yang, B.; Zheng, F.-Y.,On Curvature Tensors of Hermitian Manifolds. arXiv:1602.01189.

[35] Yau, S.-T., On the Ricci curvature of a compact Kähler manifold and the complex Monge-Ampère equation, I, Comm. Pure Appl. Math. 31 (1978), no.3, 339411.

[36] Yu, C., Hessian Comparison and Spectrum Lower Bound of Almost Hermitian Manifolds, Chin. Ann. Math. Ser. B, 39 (2018), no. 4, 755-772.

(Man-Chun Lee) Department of Mathematics, Northwestern University, Evanston, IL 60208, USA

E-mail address: mclee@math.northwestern.edu 